\title{
RETIFICADORES PWM TRIFÁSICOS UNIDIRECIONAIS COM ALTO FATOR DE POTÊNCIA
}

\author{
Ivo Barbi, Yales Rômulo de Novaes, Fabiana Pöttker de Souza e Deivis Borgonovo \\ INEP-UFSC \\ Caixa Postal 5119 \\ 88040-970 - Florianópolis - SC \\ Brasil
}

\begin{abstract}
Resumo - Este artigo apresenta algumas das principais topologias de retificadores PWM trifásicos sem neutro encontradas na literatura, bem como uma breve comparação entre elas. Na sequência apresenta-se uma modelagem genérica para estes retificadores, com modelo completo e simplificado, para projetar tanto as malhas de corrente, quanto a(s) de tensão. Mostra-se então uma metodologia e exemplo de projeto. Finalmente são apresentados os resultados experimentais de um protótipo de $6 \mathrm{~kW}$ com saída em 2 níveis e outro de $26 \mathrm{~kW}$ com saída em 3 níveis.
\end{abstract}

Abstract - This paper presents some topologies of three-phase three wire PWM rectifiers and a comparison among them. A generic modeling is also presented with a complete and a simplified model, for designing the voltages and current control loops. Design methodologies are suggested as well as design procedure. Experimental results for a $6 \mathrm{~kW}$ two level rectifier and for a $26 \mathrm{~kW}$ three level rectifier validates the analysis.

\section{I - INTRODUÇÃO}

É sabido que a distribuição de energia elétrica é feita, exceto em raras exceções, em corrente alternada. No entanto, sabe-se também que para muitas aplicações, principalmente para a alimentação de equipamentos eletrônicos, necessita-se desta energia disponível em corrente contínua.

Desta forma, da necessidade de se converter corrente alternada - $\mathrm{CA}$ - em corrente contínua - $\mathrm{CC}$-, surgiram os conversores $\mathrm{CA}-\mathrm{CC}$, ou simplesmente retificadores. Além disso, para níveis elevados de potência, geralmente acima de alguns quilowatts, se faz necessária a utilização de retificadores trifásicos, para garantir o equilíbrio de potência entre as fases.

Assim, quando se tem acesso ao neutro, pode-se utilizar por exemplo, três retificadores monofásicos, um para cada fase. No entanto, como nem sempre dispõe-se de neutro, ou quando sua presença é indesejável, esta solução deixa de ser factível. Surge então a necessidade de se utilizar retificadores trifásicos sem neutro.

As fontes de alimentação trifásicas convencionais, utilizam retificadores a diodo, ou a tiristores quando se deseja algum controle do fluxo de potência e da tensão de saída. No entanto, as características de entrada destes retificadores criam problemas para a rede comercial de energia elétrica, dentre os quais podem ser destacados:

- Distorção harmônica das correntes de entrada, com consequente redução do fator de potência;

- Distorção nas tensões da rede de alimentação, devido à circulação das componentes harmônicas das correntes através das impedâncias de linha, o que pode comprometer o bom funcionamento de outros equipamentos conectados à mesma rede;

- Aumento das perdas nos elementos das redes de transmissão e distribuição;

- Necessidade de geração de grandes quantidades de potência reativa, elevando os custos de todo o sistema;

- Diminuição do rendimento da estrutura, devido ao elevado valor eficaz das correntes;

- Interferência eletromagnética em sinais de controle e comunicação, como por exemplo em sistemas de telecomunicações, entre outros.

Desta forma, devido aos problemas citados, geralmente as normas e regulamentações internacionais para sistemas de telecomunicações são tomadas como referência nas pesquisas nesta área, por serem bastante rígidas.

Além disso, ao contrário dos retificadores PWM monofásicos com fator de potência unitário, que utilizam uma ponte completa de diodos e um conversor boost, para os retificadores $\mathrm{PWM}$ trifásicos sem neutro não existe uma solução consagrada que possa ser tomada como referência.

Assim, são apresentadas algumas topologias com saída em 2 e 3 níveis. Será também apresentada a modelagem dos conversores, necessária para projetar as malhas de controle de tensão e corrente, seguida de procedimentos de projeto e resultados experimentais de um retificador 2 níveis de $6 \mathrm{~kW}$ e de um retificador 3 níveis de $26 \mathrm{~kW}$, ambos com fator de potência unitário.

\section{II - TOPOLOGIAS}

Um dos fatores determinantes para a escolha da topologia do retificador é a tensão da rede e do barramento CC. Para tensões elevadas, as topologias a três níveis são mais indicadas porque a tensão sobre as chaves corresponde à metade da tensão total no barramento CC. Já para as topologias a dois níveis a tensão nas chaves é a própria tensão de saída.

As topologias do tipo BUCK são desconsideradas, pois apresentam indutores de baixa freqüência na saída e necessitam de filtros de entrada volumosos. São apresentadas então apenas topologias do tipo BOOST.

\section{A. Topologias Dois Níveis}

Dentre as topologias de retificadores do tipo PWM dois níveis, tem-se a topologia que utiliza seis interruptores comandados, apresentada na Fig. 1 [1]. Esta topologia é uma das mais conhecidas na literatura, principalmente por ser muito utilizada operando como inversor trifásico [2].

Esta topologia possui como característica principal a bidirecionalidade no fluxo de potência e, como a maioria das 
topologias do tipo elevadoras, a tensão de saída deve ser maior do que o valor máximo de pico da tensão de linha. Esta topologia, como todas que serão apresentadas neste artigo, propicia uma reduzida taxa de distorção harmônica da corrente de entrada, já que utiliza as técnicas ativas para controlar estas correntes. Entretanto, devido à sua configuração, exige maior esforço na concepção dos circuitos de comando e controle, exibindo como maior desvantagem a possibilidade de curto-circuito do barramento CC. Outra desvantagem desta topologia está relacionada ao custo. Em função da aplicação, a bidirecionalidade de energia pode se tornar uma característica dispensável, como nas aplicações em fontes para telecomunições.

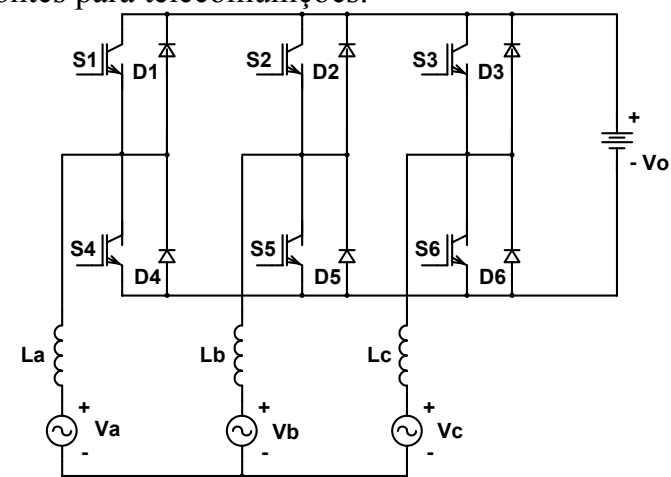

Fig. 1 - Retificador Boost dois níveis bidirecional - Boost 2n-01.

$\mathrm{Na}$ Fig. 2 apresenta-se outra topologia de retificador trifásico a dois níveis. Esta topologia é uma excelente candidata para as aplicações do tipo dois níveis e sua concepção foi baseada no inversor NPC [3], entretanto sofreu simplificações, pois não necessita de bidirecionalidade do fluxo de energia e nem da redução da tensão sobre os interruptores. O comando dos interruptores é de fácil implementação, já que ambos de um mesmo braço podem ser comandados de maneira concomitante. Cabe observar que, caso sejam utilizados interruptores do tipo MOSFET, os diodos em anti-paralelo com os interruptores podem ser os próprios diodos intrínsecos ao componente, reduzindo a complexidade construtiva.

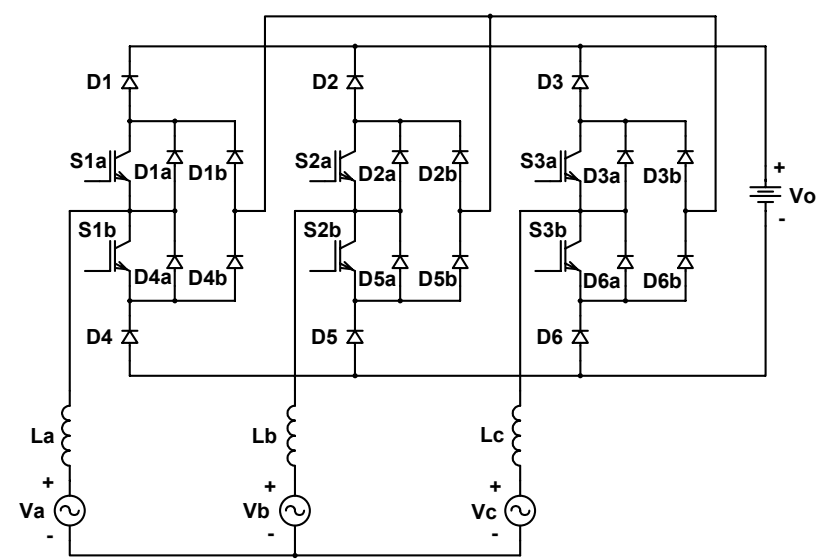

Fig. 2 - Retificador PWM unidirecional trifásico dois níveis Boost 2n-02.

Derivada da topologia apresentada em [4], tem-se a topologia Boost 2n-03, apresentada na Fig. 3. Observa-se que apesar do número de indutores ser maior, a indutância necessária para se obter a mesma ondulação de corrente da topologia Boost 2n - 02 é a metade. Esta topologia também é uma excelente candidata para as aplicações de baixa tensão de entrada.

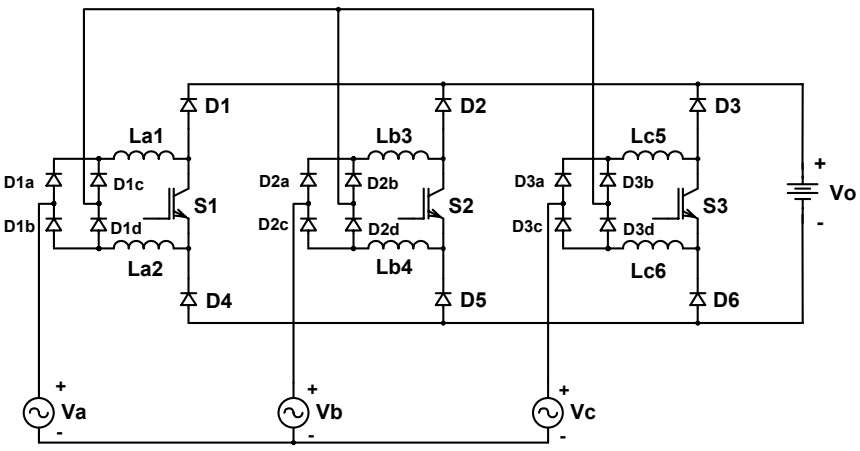

Fig. 3 - Retificador PWM unidirecional trifásico dois níveis Boost 2n - 03.

Com características muito similares à topologia Boost $2 \mathrm{n}$ 02, tem-se a topologia apresentada na Fig. 4, aqui denominada de Boost 2n-04, derivada de [6]. Esta topologia utiliza apenas um interruptor controlável por fase, reduzindo à metade a necessidade de circuitos de comando isolados, em relação às estruturas Boost 2n-01 e 2n-02.

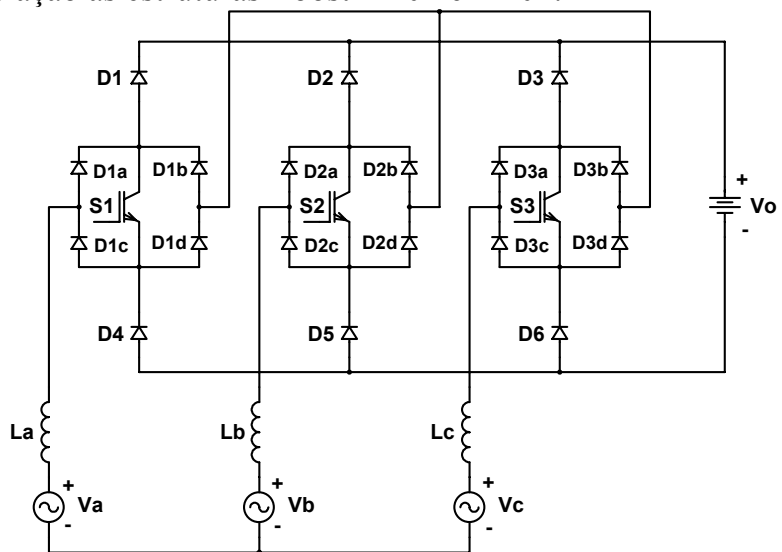

Fig. 4 - Retificador PWM unidirecional trifásico dois níveis Boost 2n - 04.

Havendo a necessidade de se escolher uma destas três topologias, sendo que a primeira já havia sido descartada devido às questões já apresentadas, optou-se pela investigação através de simulação das três últimas topologias descritas.

Principalmente, pelo reduzido número de componentes, e pela simplicidade de realização dos circuitos de controle e comando, optou-se pela topologia Boost 2 n - 04 para fazer parte do projeto de uma unidade retificadora para telecomunicações. Os resultados experimentais doravante apresentados são relativos a implementação desta topologia.

\section{B. Topologias Três Niveis}

As topologias três níveis possuem dois capacitores conectados no barramento $\mathrm{CC}$, cujo ponto central é então conectado a um ponto comum das três fases. Com uma estratégia de controle apropriada garante-se a divisão equitativa da tensão nos capacitores do barramento. Desta forma, a tensão aplicada sobre os interruptores é a metade da tensão total do barramento $\mathrm{CC}\left(\mathrm{V}_{\mathrm{o}} / 2\right)$, tornando estas topologias atrativas para aplicações com tensão de entrada elevada.

O retificador PWM com grampeamento do ponto neutro apresentado na Fig. 5 tem como característica principal a 
bidirecionalidade do fluxo de potência [3]. Esta topologia além de ser complexa e de elevado custo também apresenta possibilidade de curto de braço. Portanto não será incluída na análise.

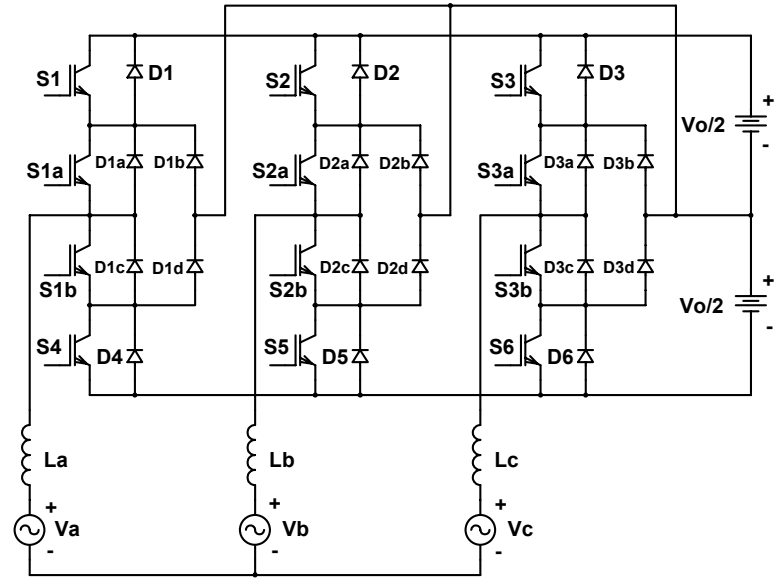

Fig. 5 - Retificador PWM bidirecional trifásico três níveis.

Retirando-se os interruptores $\mathrm{S}_{1}, \mathrm{~S}_{2}, \mathrm{~S}_{3}, \mathrm{~S}_{4}, \mathrm{~S}_{5}$ e $\mathrm{S}_{6}$, do retificador da Fig. 5, obtém-se uma topologia mais simples, unidirecional, e sem a possibilidade de curto de braço, sendo portanto uma excelente candidata para aplicações com alta tensão de entrada. Esta topologia é apresentada na Fig. 6. Os dois interruptores de cada braço são acionados com o mesmo sinal de comando. No entanto, os circuitos de comando de cada fase devem ser isolados. Os diodos em anti-paralelo com os interruptores podem ser os próprios diodos intrínsecos do MOSFET.

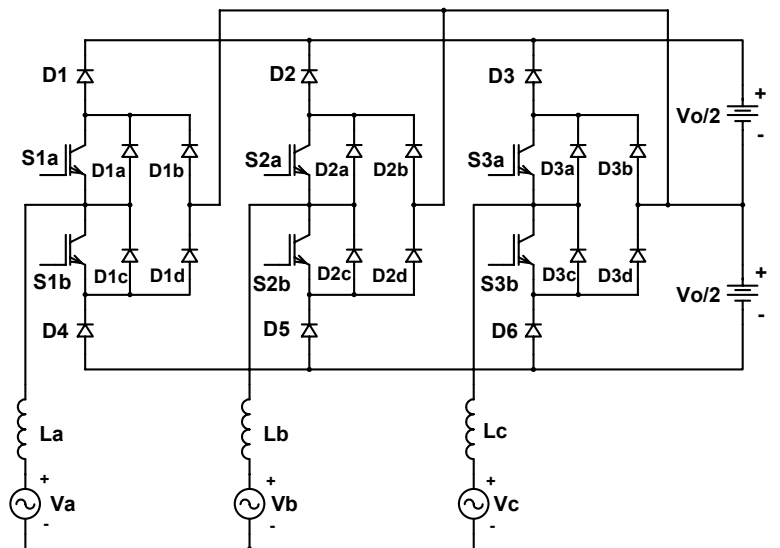

Fig. 6 - Retificador PWM unidirecional trifásico três níveis Boost 3n-02.

A topologia apresentada na Fig. 7 apresenta um número de diodos menor que a topologia Boost $3 n-02$, e os sinais de comando também são os mesmos para cada fase [5]. Esta topologia também é candidata para aplicações de alta tensão.

A topologia apresentada na Fig. 8 é outra opção para tensões elevadas [6]. Por apresentar apenas um interruptor comandável por fase, tem-se a metade dos circuitos de comando isolados, comparando-se com as demais topologias.

A topologia apresentada na Fig. 9 é outra opção para tensões elevadas [7], no entanto, apresenta a tensão total do barramento aplicada aos diodos da ponte retificadora $\left(\mathrm{D}_{1}, \mathrm{D}_{2}\right.$, $\left.\mathrm{D}_{3}, \mathrm{D}_{4}, \mathrm{D}_{5}, \mathrm{D}_{6}\right)$, se tornando menos atrativa que as demais topologias.

\footnotetext{
${ }^{1}$ Este conversor está sendo patenteado pela empresa TYCO Electronics
}

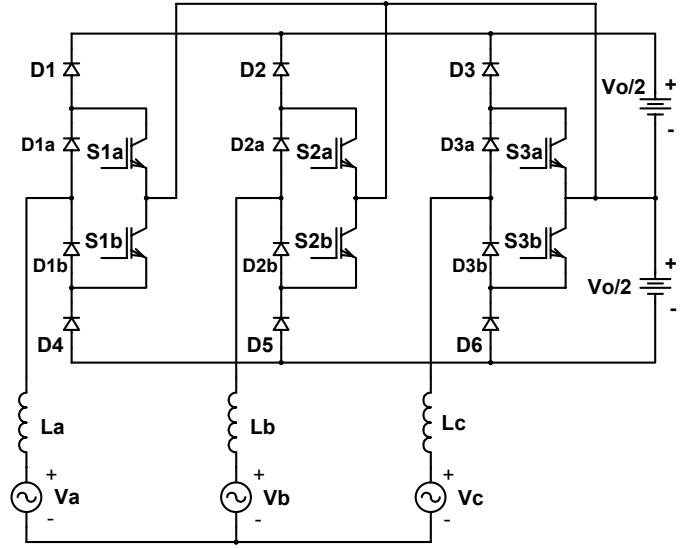

Fig. 7 - Retificador PWM unidirecional trifásico três níveis Boost 3n-03.

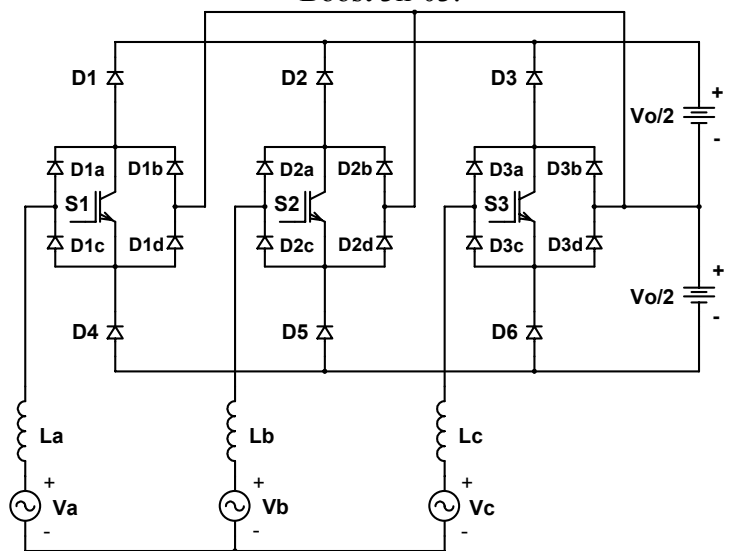

Fig. 8 - Retificador PWM unidirecional trifásico três níveis Boost 3n-04.

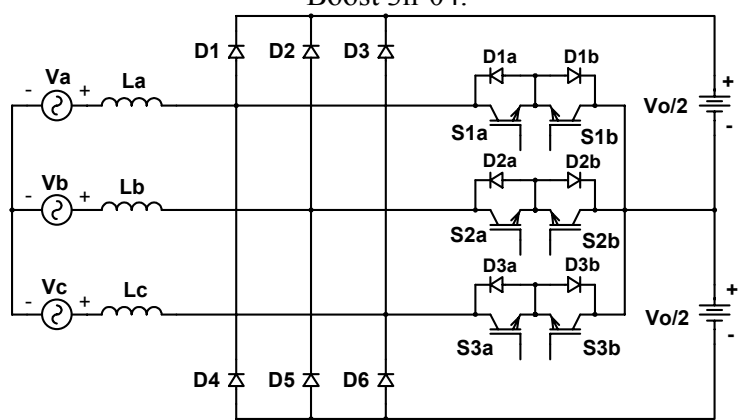

Fig. 9 - Retificador PWM unidirecional trifásico três níveis Boost 3n- 05 .

Através de simulação das quatro topologias apresentadas nas Figs. 6 a 9, verificou-se que as topologias Boost 3n-02 e Boost 3n-05 apresentam perdas menores nos semicondutores. Assim, pelas reduzidas perdas nos semicondutores e principalmente por ser uma topologia nova, optou-se pela Boost 3n-02 para fazer parte do projeto de uma unidade retificadora para telecomunicações [8]. O exemplo de projeto e os resultados experimentais apresentados referem-se a esta topologia escolhida.

\section{III - MODELAGEM E DEFINIÇÃO DOS CONTROLADORES}

Seria de grande interesse a obtenção de uma metodologia rápida e simples, porém eficiente e confiável, para analisar e modelar os retificadores PWM trifásicos, principalmente as novas topologias.

Desta forma, será apresentado o desenvolvimento da metodologia para analisar e modelar tais conversores, 
baseada no conversor bidirecional 2 níveis mais simples, apresentado na Fig.1, podendo-se então estender a metodologia para os demais conversores, inclusive para os 3 níveis.

\section{A. Obtenção do Modelo do Conversor a Partir do Lado CA}

Assim sendo, para obter o modelo visto a partir do lado CA, utilizado para controlar as correntes de entrada do retificador, será apresentada a elaboração de uma metodologia para análise, modelagem e controle, inicialmente aplicada para o conversor da Fig.1, para então na sequência generalizar a análise.

Deve-se ressaltar ainda que o modelo genérico visto a partir da entrada é o mesmo para conversores 2 e 3 níveis, como será provado mais adiante.

Pode ser observado que o circuito a ser analisado é tradicionalmente utilizado como conversor CC-CA. A metodologia que será apresentada também pode ser utilizada no controle de inversores trifásicos ou filtros ativos.

O circuito do conversor apresentado na Fig.1, pode ser representado pelo circuito simplificado da Fig. 10, sem perda de generalidade [9]:

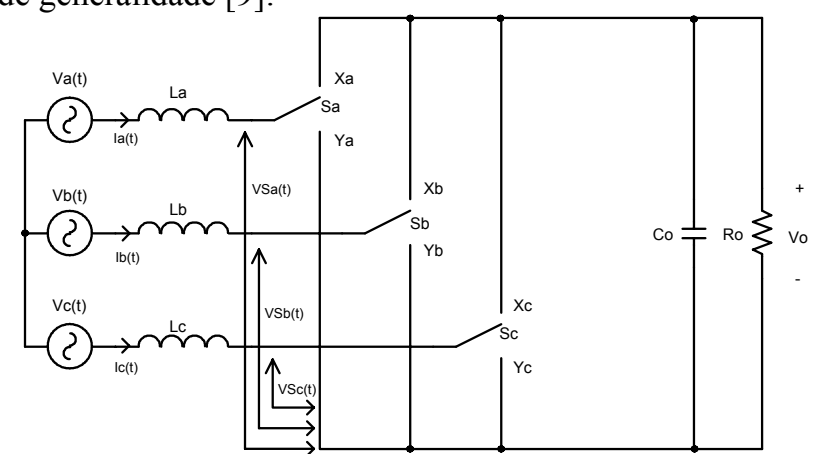

Fig. 10 - Circuito simplificado do conversor CA-CC trifásico bidirecional apresentado na Fig.1.

Assim, a partir do circuito apresentado na Fig.10, pode-se observar que quando o interruptor $S_{a}$ está na posição $X_{a}$, temse $\mathrm{V}_{\mathrm{Sa}}(\mathrm{t})=\mathrm{V}_{\mathrm{o}}$ e quando $\mathrm{S}_{\mathrm{a}}$ está na posição $\mathrm{Y}_{\mathrm{a}}$ tem-se $\mathrm{V}_{\mathrm{Sa}}(\mathrm{t})=$ 0 , a partir desta observação e seguindo o mesmo raciocínio para os interruptores $S_{b}$ e $S_{c}$, pode-se escrever:

$$
\left\{\begin{array}{l}
V_{S a}(t)=\left[1-D_{a}(t)\right] \cdot V_{o} \\
V_{S b}(t)=\left[1-D_{b}(t)\right] \cdot V_{o} \\
V_{S c}(t)=\left[1-D_{c}(t)\right] \cdot V_{o}
\end{array}\right.
$$

onde:

$$
\left\{\begin{array}{l}
\mathrm{Da}(\mathrm{t})= \begin{cases}0, & \mathrm{Sa} \rightarrow \mathrm{Xa} \\
1, & \mathrm{Sa} \rightarrow \mathrm{Ya}\end{cases} \\
\mathrm{Db}(\mathrm{t})= \begin{cases}0, & \mathrm{Sb} \rightarrow \mathrm{Xb} \\
1, & \mathrm{Sb} \rightarrow \mathrm{Yb}\end{cases} \\
\mathrm{Dc}(\mathrm{t})= \begin{cases}0, & \mathrm{Sc} \rightarrow \mathrm{Xc} \\
1, & \mathrm{Sc} \rightarrow \mathrm{Yc}\end{cases}
\end{array}\right.
$$

Pode-se ainda representar o conversor apresentado na Fig. 10, pelo circuito equivalente apresentado na Fig.11.

Além disso, $\mathrm{V}_{\mathrm{O}}$ corresponde à tensão de saída, que por enquanto será considerada constante.

Observa-se ainda que o circuito equivalente da Fig. 11 apresenta três variáveis de estado (correntes nos indutores), o que daria origem a um sistema de terceira ordem, entretanto, como o sistema não apresenta neutro, surge uma restrição, que é definida por (3):

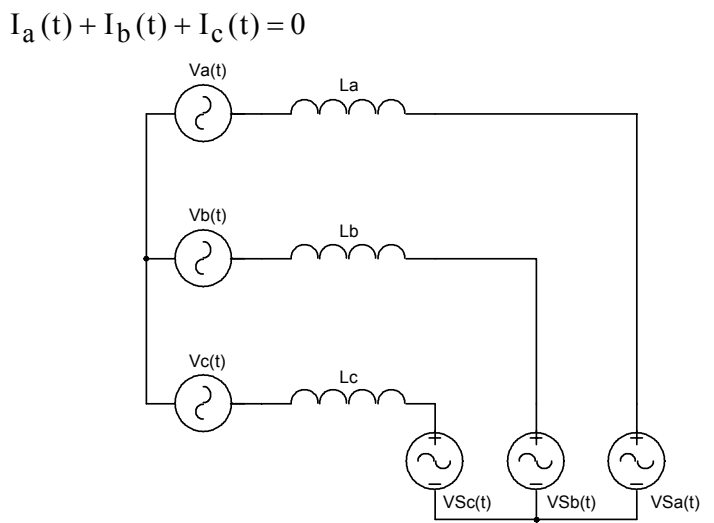

Fig. 11 - Circuito equivalente ao conversor apresentado na Fig. 10.

Ou seja, tem-se um sistema apenas de segunda ordem, com duas variáveis de estado independentes, já que a terceira é definida como uma combinação linear das outras duas.

Observando então a Fig.11, pode-se escrever:

$$
\left\{\begin{array}{l}
{\left[\mathrm{V}_{\mathrm{a}}(\mathrm{t})-\mathrm{V}_{\mathrm{b}}(\mathrm{t})\right]=\left[\mathrm{V}_{\mathrm{La}}(\mathrm{t})-\mathrm{V}_{\mathrm{Lb}}(\mathrm{t})\right]+\left[\mathrm{V}_{\mathrm{Sa}}(\mathrm{t})-\mathrm{V}_{\mathrm{Sb}}(\mathrm{t})\right]} \\
{\left[\mathrm{V}_{\mathrm{b}}(\mathrm{t})-\mathrm{V}_{\mathrm{c}}(\mathrm{t})\right]=\left[\mathrm{V}_{\mathrm{Lb}}(\mathrm{t})-\mathrm{V}_{\mathrm{Lc}}(\mathrm{t})\right]+\left[\mathrm{V}_{\mathrm{Sb}}(\mathrm{t})-\mathrm{V}_{\mathrm{Sc}}(\mathrm{t})\right]} \\
{\left[\mathrm{V}_{\mathrm{c}}(\mathrm{t})-\mathrm{V}_{\mathrm{a}}(\mathrm{t})\right]=\left[\mathrm{V}_{\mathrm{Lc}}(\mathrm{t})-\mathrm{V}_{\mathrm{La}}(\mathrm{t})\right]+\left[\mathrm{V}_{\mathrm{Sc}}(\mathrm{t})-\mathrm{V}_{\mathrm{Sa}}(\mathrm{t})\right]}
\end{array}\right.
$$

Pode-se definir ainda:

$$
\left\{\begin{array}{l}
V_{S a b}(t)=V_{S a}(t)-V_{S b}(t) \\
V_{S b c}(t)=V_{S b}(t)-V_{S c}(t) \\
V_{S c a}(t)=V_{S c}(t)-V_{S a}(t)
\end{array}\right.
$$

Onde obviamente tem-se:

$$
\mathrm{V}_{\mathrm{Sab}}(\mathrm{t})+\mathrm{V}_{\mathrm{Sbc}}(\mathrm{t})+\mathrm{V}_{\mathrm{Sca}}(\mathrm{t})=0
$$

Desta forma, obtém-se um circuito ainda mais simples para representar o modelo do conversor visto a partir do lado CA, que é apresentado na Fig.12. Pode-se escrever então:

$$
\left\{\begin{array}{l}
{\left[\mathrm{V}_{\mathrm{a}}(\mathrm{t})-\mathrm{V}_{\mathrm{b}}(\mathrm{t})\right]=\left[\mathrm{V}_{\mathrm{La}}(\mathrm{t})-\mathrm{V}_{\mathrm{Lb}}(\mathrm{t})\right]+\mathrm{V}_{\mathrm{Sab}}(\mathrm{t})} \\
{\left[\mathrm{V}_{\mathrm{b}}(\mathrm{t})-\mathrm{V}_{\mathrm{c}}(\mathrm{t})\right]=\left[\mathrm{V}_{\mathrm{Lb}}(\mathrm{t})-\mathrm{V}_{\mathrm{Lc}}(\mathrm{t})\right]+\mathrm{V}_{\mathrm{Sbc}}(\mathrm{t})} \\
{\left[\mathrm{V}_{\mathrm{c}}(\mathrm{t})-\mathrm{V}_{\mathrm{a}}(\mathrm{t})\right]=\left[\mathrm{V}_{\mathrm{Lc}}(\mathrm{t})-\mathrm{V}_{\mathrm{La}}(\mathrm{t})\right]+\mathrm{V}_{\mathrm{Sca}}(\mathrm{t})(\text { Redundante) }}
\end{array}\right.
$$

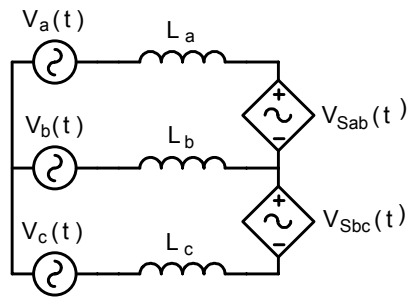

Fig. 12 - Circuito equivalente ao apresentado na Fig. 11.

Considerando ainda que $\mathrm{L}_{\mathrm{a}}=\mathrm{L}_{\mathrm{b}}=\mathrm{L}_{\mathrm{c}}=\mathrm{L}$, pode-se escrever:

$$
\left\{\begin{array}{l}
V_{L a}(t)-V_{L b}(t)=L \cdot \frac{d I_{a}(t)}{d t}-L \cdot \frac{d I_{b}(t)}{d t}=L \cdot \frac{d}{d t}\left[I_{a}(t)-I_{b}(t)\right]=L \cdot \frac{d I_{a b}(t)}{d t}(8) \\
V_{L b}(t)-V_{L c}(t)=L \cdot \frac{d I_{b}(t)}{d t}-L \cdot \frac{d I_{c}(t)}{d t}=L \cdot \frac{d}{d t}\left[I_{b}(t)-I_{c}(t)\right]=L \cdot \frac{d I_{b c}(t)}{d t} \\
V_{L c}(t)-V_{L a}(t)=L \cdot \frac{d I_{c}(t)}{d t}-L \cdot \frac{d I_{a}(t)}{d t}=L \cdot \frac{d}{d t}\left[I_{c}(t)-I_{a}(t)\right]=L \cdot \frac{d I_{c a}(t)}{d t}
\end{array}\right.
$$

Tem-se então: 


$$
\left\{\begin{array}{l}
V_{a b}(t)=L \frac{d I_{a b}(t)}{d t}-V_{o} \cdot D_{a b}(t) \\
V_{b c}(t)=L \frac{d I_{b c}(t)}{d t}-V_{o} \cdot D_{b c}(t) \\
V_{c a}(t)=L \frac{d I_{c a}(t)}{d t}-V_{o} \cdot D_{c a}(t)
\end{array}\right.
$$

Pode-se observar novamente que $\mathrm{I}_{\mathrm{ab}}(\mathrm{t})+\mathrm{I}_{\mathrm{bc}}(\mathrm{t})+\mathrm{I}_{\mathrm{ca}}(\mathrm{t})=0$, ou seja, umas das correntes fictícias pode ser escrita como uma combinação linear das outras duas, concluindo-se então que se pode controlar, de forma independente, duas combinações linearmente independentes das correntes de entrada, o que é suficiente para garantir o formato senoidal das três correntes de entrada.

Observa-se no entanto, que as razões cíclicas $D_{a b}(t), D_{b c}(t)$ e $D_{c a}(t)$ são obviamente fictícias, sendo elas o resultado de combinações das razões cíclicas reais $D_{a}(t), D_{b}(t)$ e $D_{c}(t)$. No entanto, a partir das razões cíclicas fictícias pode-se facilmente determinar as razões cíclicas reais. Todavia devese ter em mente que esta metodologia de controle somente seria factível utilizando processadores digitais de sinal (DSP), utilizando controle digital.

\section{B. Obtenção da Função de Transferência Simplificada}

Pode-se ainda obter um modelo simplificado de pequenos sinais, onde se poderia supostamente controlar de forma independente as três correntes de entrada. Desta forma, da Fig. 11, tem-se:

$$
\begin{aligned}
& V_{a}(t)-V_{L a}(t)-V_{S a}(t)=V_{b}(t)-V_{L b}(t)-V_{S b}(t)= \\
& V_{c}(t)-V_{L c}(t)-V_{S c}(t)
\end{aligned}
$$

Logo, a soma das três expressões é igual ao triplo de uma delas:

$$
\begin{aligned}
& 3 \cdot\left[\mathrm{V}_{\mathrm{a}}(\mathrm{t})-\mathrm{V}_{\mathrm{La}}(\mathrm{t})-\mathrm{V}_{\mathrm{Sa}}(\mathrm{t})\right]=\left[\mathrm{V}_{\mathrm{a}}(\mathrm{t})+\mathrm{V}_{\mathrm{b}}(\mathrm{t})+\mathrm{V}_{\mathrm{c}}(\mathrm{t})\right]- \\
& {\left[\mathrm{V}_{\mathrm{La}}(\mathrm{t})+\mathrm{V}_{\mathrm{Lb}}(\mathrm{t})+\mathrm{V}_{\mathrm{Lc}}(\mathrm{t})\right]-\left[\mathrm{V}_{\mathrm{Sa}}(\mathrm{t})+\mathrm{V}_{\mathrm{Sb}}(\mathrm{t})+\mathrm{V}_{\mathrm{Sc}}(\mathrm{t})\right]}
\end{aligned}
$$

Além disso, considerando-se que a alimentação seja perfeitamente senoidal e equilibrada, lembrando que não há presença de neutro e tendo $\mathrm{L}_{\mathrm{a}}=\mathrm{L}_{\mathrm{b}}=\mathrm{L}_{\mathrm{c}}$ pode-se afirmar que:

$$
\begin{aligned}
& V_{a}(t)+V_{b}(t)+V_{c}(t)=0 \\
& I_{L a}(t)+I_{L b}(t)+I_{L c}(t)=0 \Rightarrow V_{L a}(t)+V_{L b}(t)+V_{L c}(t)=0
\end{aligned}
$$

Obtém-se então:

3. $\left[\mathrm{V}_{\mathrm{a}}(\mathrm{t})-\mathrm{V}_{\mathrm{La}}(\mathrm{t})-\mathrm{V}_{\mathrm{Sa}}(\mathrm{t})\right]+\left[\mathrm{V}_{\mathrm{Sa}}(\mathrm{t})+\mathrm{V}_{\mathrm{Sb}}(\mathrm{t})+\mathrm{V}_{\mathrm{Sc}}(\mathrm{t})\right]=0$

Logo:

$$
3 V_{a}(t)-3 L \frac{d I_{L a}(t)}{d t}-2 V_{S a}(t)+V_{S b}(t)+V_{S c}(t)=0
$$

Ou:

$$
3 \cdot V_{a}(t)-3 \cdot L \cdot \frac{d I_{L a}(t)}{d t}+V_{o} \cdot\left\{\begin{array}{l}
-2\left[1-D_{a}(t)\right]+\left[1-D_{b}(t)\right] \\
+\left[1-D_{c}(t)\right]
\end{array}\right\}=0
$$

A partir de (16), aplica-se um modelo de pequenos sinais, para um curto intervalo de tempo, onde a tensão de alimentação $\mathrm{V}_{\mathrm{a}}(\mathrm{t})$ pode ser considerada constante. Além disso, será inserida neste ponto a simplificação desta análise, considerando-se que $\mathrm{D}_{\mathrm{b}}(\mathrm{t})$ e $\mathrm{D}_{\mathrm{c}}(\mathrm{t})$ (ou a soma delas) permanece constante, de forma que se obtém então:

$$
\begin{aligned}
& 3 \cdot 0-3 \cdot \mathrm{L} \cdot \frac{\mathrm{di}_{\mathrm{La}}(\mathrm{t})}{\mathrm{dt}}+\mathrm{V}_{\mathrm{o}} \cdot\left\{\begin{array}{l}
-2\left[0-\mathrm{d}_{\mathrm{a}}(\mathrm{t})\right]+[0-0] \\
+[0-0]
\end{array}\right\}=0 \\
& -3 \cdot \mathrm{L} \cdot \frac{\mathrm{di}_{\mathrm{La}}(\mathrm{t})}{\mathrm{dt}}+\mathrm{V}_{\mathrm{o}} \cdot 2 \cdot \mathrm{d}_{\mathrm{a}}(\mathrm{t})=0 \\
& \text { Aplicando a transformada de Laplace: } \\
& 3 \cdot \mathrm{L} \cdot \mathrm{s} \cdot \mathrm{i}_{\mathrm{La}}(\mathrm{s})=\mathrm{V}_{\mathrm{o}} \cdot 2 \cdot \mathrm{d}_{\mathrm{a}}(\mathrm{s})
\end{aligned}
$$

De forma análoga, obtém-se o mesmo resultado para as fases "b" e "c", obtendo-se então a função de transferência:

$$
\frac{\mathrm{i}(\mathrm{s})}{\mathrm{d}(\mathrm{s})}=\frac{\mathrm{V}_{\mathrm{o}}}{\mathrm{s}\left(\frac{3}{2} \mathrm{~L}\right)}
$$

O resultado obtido em (20) corresponde à própria função de transferência do conversor Boost CC-CC convencional, a não ser pelo fato de apresentar uma indutância equivalente $50 \%$ maior que a indutância física.

Deve-se observar que para conversores 2 níveis, $V_{O}$ representa a tensão total de saída, enquanto que para conversores 3 níveis, $\mathrm{V}_{\mathrm{O}}$ representa a tensão de saída apenas em um dos barramentos, de forma que se pode considerar $V_{O}$ como metade da tensão total de saída.

Com esta função de transferência simplificada pode-se projetar os controladores de corrente e obter bons resultados práticos, no entanto deve-se ter ciência de que esta simplificação pode acarretar alguns problemas, como deformações nas correntes de entrada, pois se está desprezando o acoplamento entre as funções de transferência.

$\mathrm{Na}$ verdade os controladores não atuarão realmente de forma independente, mas irão sim interagir.

C. Obtenção do Modelo do Conversor a Partir da Saída CC Sejam as tensões de alimentação definidas por:

$\left\{\begin{array}{l}V_{a}(t)=V_{P} \cdot \operatorname{sen}(\omega t) \\ V_{b}(t)=V_{P} \cdot \operatorname{sen}\left(\omega t+120^{\circ}\right) \\ V_{c}(t)=V_{P} \cdot \operatorname{sen}\left(\omega t-120^{\circ}\right)\end{array}\right.$

Tem-se então:

$$
\left\{\begin{array}{l}
\mathrm{V}_{\mathrm{ab}}(\mathrm{t})=\sqrt{3} \cdot \mathrm{V}_{\mathrm{P}} \cdot \operatorname{sen}\left(\omega \mathrm{t}-30^{\circ}\right) \\
\mathrm{V}_{\mathrm{bc}}(\mathrm{t})=\sqrt{3} \cdot \mathrm{V}_{\mathrm{P}} \cdot \operatorname{sen}\left(\omega \mathrm{t}+90^{\circ}\right) \\
\mathrm{V}_{\mathrm{ca}}(\mathrm{t})=\sqrt{3} \cdot \mathrm{V}_{\mathrm{P}} \cdot \operatorname{sen}\left(\omega \mathrm{t}-150^{\circ}\right)
\end{array}\right.
$$

Considera-se ainda que os controladores de corrente garantem seu formato senoidal, sem defasamento em relação às tensões de alimentação, desta forma tem-se:

$$
\left\{\begin{array}{l}
I_{a}(t)=I_{P} \cdot \operatorname{sen}(\omega t) \\
I_{b}(t)=I_{P} \cdot \operatorname{sen}\left(\omega t+120^{\circ}\right) \\
I_{c}(t)=I_{P} \cdot \operatorname{sen}\left(\omega t-120^{\circ}\right)
\end{array}\right.
$$

Assim sendo, pode-se definir a potência média de entrada por:

$$
\mathrm{P}_{\mathrm{IN}}=\frac{\mathrm{P}_{\mathrm{o}}}{\eta}=\frac{3}{2} \cdot \mathrm{V}_{\mathrm{P}} \cdot \mathrm{I}_{\mathrm{P}}
$$

Onde:

$\mathrm{P}_{\mathrm{IN}} \rightarrow$ Potência média de entrada;

$\mathrm{P}_{\mathrm{O}} \rightarrow$ Potência média de saída;

$\eta \rightarrow$ Rendimento total da unidade retificadora; Logo: 


$$
I_{P}=\frac{2 \cdot P_{o}}{3 \cdot \eta \cdot V_{P}}
$$

Pode-se escrever ainda:

$$
\left\{\begin{array}{l}
I_{a b}(t)=\frac{2 \cdot P_{o}}{\sqrt{3} \cdot \eta \cdot V_{P}} \cdot \operatorname{sen}\left(\omega t-30^{\circ}\right) \\
I_{b c}(t)=\frac{2 \cdot P_{o}}{\sqrt{3} \cdot \eta \cdot V_{P}} \cdot \operatorname{sen}\left(\omega t+90^{\circ}\right) \\
I_{c a}(t)=\frac{2 \cdot P_{o}}{\sqrt{3} \cdot \eta \cdot V_{P}} \cdot \operatorname{sen}\left(\omega t-150^{\circ}\right)
\end{array}\right.
$$

A partir da Fig. 12, tem-se:

$$
\left\{\begin{array}{l}
V_{a b}(t)=L \cdot \frac{d I_{a b}(t)}{d t}+V_{S a b}(t) \\
V_{b c}(t)=L \cdot \frac{d I_{b c}(t)}{d t}+V_{S b c}(t) \\
V_{c a}(t)=L \cdot \frac{d I_{c a}(t)}{d t}+V_{S c a}(t)
\end{array}\right.
$$

Logo:

$$
\left\{\begin{array}{l}
D_{a b}(t)=\frac{V_{a b}(t)-L \cdot \frac{d I_{a b}(t)}{d t}}{V_{o}} \\
D_{b c}(t)=\frac{V_{b c}(t)-L \cdot \frac{d I_{b c}(t)}{d t}}{V_{o}} \\
D_{c a}(t)=\frac{V_{c a}(t)-L \cdot \frac{d I_{c a}(t)}{d t}}{V_{o}}
\end{array}\right.
$$

Desta forma, substituindo-se (26) e (27) em (28), obtémse:

$\left\{\begin{array}{l}D_{a b}(t)=\frac{\sqrt{3} \cdot V_{P}}{V_{o}} \cdot \operatorname{sen}\left(\omega t-30^{\circ}\right)-\frac{\omega \cdot L \cdot 2 \cdot P_{o}}{\sqrt{3} \cdot \eta \cdot V_{P} \cdot V_{o}} \cdot \cos \left(\omega t-30^{\circ}\right) \\ D_{b c}(t)=\frac{\sqrt{3} \cdot V_{P}}{V_{o}} \cdot \operatorname{sen}\left(\omega t+90^{\circ}\right)-\frac{\omega \cdot L \cdot 2 \cdot P_{o}}{\sqrt{3} \cdot \eta \cdot V_{P} \cdot V_{o}} \cdot \cos \left(\omega t+90^{\circ}\right) \\ D_{c a}(t)=\frac{\sqrt{3} \cdot V_{P}}{V_{o}} \cdot \operatorname{sen}\left(\omega t-150^{\circ}\right)-\frac{\omega \cdot L \cdot 2 \cdot P_{o}}{\sqrt{3} \cdot \eta \cdot V_{P} \cdot V_{o}} \cdot \cos \left(\omega t-150^{\circ}\right)\end{array}\right.$

Pode-se simplificar ainda (29):

$$
\left\{\begin{array}{l}
D_{a b}(t)=\frac{\sqrt{3} \cdot V_{P}}{V_{o}}\left[\operatorname{sen}\left(\omega t-30^{\circ}\right)-\frac{2 \cdot \omega \cdot L \cdot P_{o}}{3 \cdot \eta \cdot V_{p}^{2}} \cdot \cos \left(\omega t-30^{\circ}\right)\right] \\
D_{b c}(t)=\frac{\sqrt{3} \cdot V_{P}}{V_{o}}\left[\operatorname{sen}\left(\omega t+90^{\circ}\right)-\frac{2 \cdot \omega \cdot L \cdot P_{o}}{3 \cdot \eta \cdot V_{p}^{2}} \cdot \cos \left(\omega t+90^{\circ}\right)\right] \\
D_{c a}(t)=\frac{\sqrt{3} \cdot V_{P}}{V_{o}}\left[\operatorname{sen}\left(\omega t-150^{\circ}\right)-\frac{2 \cdot \omega \cdot L \cdot P_{o}}{3 \cdot \eta \cdot V_{p}{ }^{2}} \cdot \cos \left(\omega t-150^{\circ}\right)\right]
\end{array}\right.
$$

Em (30) apresenta-se o comportamento das razões cíclicas, válido para qualquer instante do período da rede, para o conversor operando em regime permanente.

Pode-se observar claramente a forma como as razões cíclicas controlam diretamente o fluxo de potência ativa da alimentação para a carga, através da amplitude dos cossenos das razões cíclicas.

Ainda, a partir do circuito da Fig.10, pode-se observar que a corrente total de saída $\mathrm{I}_{\mathrm{O}}(\mathrm{t})$ é dada por:
$I_{o}(t)=I_{a}(t) \cdot\left[1-D_{a}(t)\right]+I_{b}(t) \cdot\left[1-D_{b}(t)\right]+I_{c}(t) \cdot\left[1-D_{c}(t)\right](31)$

Sabe-se ainda que:

$$
\begin{aligned}
& {\left[I_{a}(t) \cdot D_{a}(t)+I_{b}(t) \cdot D_{b}(t)+I_{c}(t) \cdot D_{c}(t)\right]=} \\
& \frac{1}{3} \cdot\left[I_{a b}(t) \cdot D_{a b}(t)+I_{b c}(t) \cdot D_{b c}(t)+I_{c a}(t) \cdot D_{c a}(t)\right]
\end{aligned}
$$

Logo, lembrando que a soma das três correntes de entrada é nula, tem-se:

$$
I_{o}(t)=-\frac{1}{3} \cdot\left[I_{a b}(t) \cdot D_{a b}(t)+I_{b c}(t) \cdot D_{b c}(t)+I_{c a}(t) \cdot D_{c a}(t)\right]
$$

Substituindo (30) e (26) em (33) e utilizando identidades trigonométricas, obtém-se:

$$
\mathrm{I}_{\mathrm{o}}(\mathrm{t})=\frac{3 \cdot \mathrm{V}_{\mathrm{P}} \cdot \mathrm{I}_{\mathrm{P}}}{2 \cdot \mathrm{V}_{\mathrm{o}}}
$$

Deve-se observar que $\mathrm{I}_{\mathrm{O}}(\mathrm{t})$, apresentada em (34), representa a corrente instantânea de saída, ou seja, desprezando as componentes de alta frequência (comutação), a corrente de saída é constante e diretamente proporcional à amplitude das correntes de entrada. Considera-se então que a malha de tensão apresente como variável de controle a amplitude das correntes de entrada, de forma que se multiplica esta amplitude pelas tensões de alimentação, para definir as referências das correntes de entrada. Tem-se então:

$$
\frac{\mathrm{I}_{\mathrm{o}}(\mathrm{s})}{\mathrm{I}_{\mathrm{P}}(\mathrm{s})}=\frac{3 \cdot \mathrm{V}_{\mathrm{P}}}{2 \cdot \mathrm{V}_{\mathrm{o}}}
$$

Além disso, considerando uma carga resistiva e um modelo simplificado dos capacitores de saída, levando em conta apenas a resistência série equivalente, tem-se:

$\mathrm{V}_{\mathrm{o}}(\mathrm{s})=\mathrm{I}_{\mathrm{o}}(\mathrm{s}) \cdot \mathrm{Z}_{\mathrm{eq}}$

Onde:

$$
\mathrm{Z}_{\mathrm{eq}}=\left[\frac{\mathrm{s} \cdot \mathrm{C}_{\mathrm{o}}}{\mathrm{s} \cdot \mathrm{R}_{\mathrm{SE}} \cdot \mathrm{C}_{\mathrm{o}}+1}+\frac{1}{\mathrm{R}_{\mathrm{eq}}}\right]^{-1}
$$

Pode-se definir ainda a resistência de carga equivalente por:

$$
\mathrm{R}_{\mathrm{o}}=\frac{\mathrm{V}_{\mathrm{o}}^{2}}{\mathrm{P}_{\mathrm{o}}}
$$

Assim, obtém-se:

$$
\frac{\mathrm{V}_{\mathrm{o}}(\mathrm{s})}{\mathrm{I}_{\mathrm{o}}(\mathrm{s})}=\frac{\frac{\mathrm{V}_{\mathrm{o}}^{2}}{\mathrm{P}_{\mathrm{o}}} \cdot\left(\mathrm{s} \cdot \mathrm{R}_{\mathrm{SE}} \cdot \mathrm{C}_{\mathrm{o}}+1\right)}{\mathrm{s} \cdot \frac{\mathrm{C}_{\mathrm{o}} \cdot \mathrm{V}_{\mathrm{o}}^{2}}{\mathrm{P}_{\mathrm{o}}}\left(1+\frac{\mathrm{R}_{\mathrm{SE}} \cdot \mathrm{P}_{\mathrm{o}}}{\mathrm{V}_{\mathrm{o}}^{2}}\right)+1}
$$

Finalmente multiplicando-se (39) por (35), obtém-se:

$$
\frac{\mathrm{V}_{\mathrm{o}}(\mathrm{s})}{\mathrm{I}_{\mathrm{o}}(\mathrm{s})} \cdot \frac{\mathrm{I}_{\mathrm{o}}(\mathrm{s})}{\mathrm{I}_{\mathrm{P}}(\mathrm{s})}=\frac{\mathrm{V}_{\mathrm{o}}(\mathrm{s})}{\mathrm{I}_{\mathrm{P}}(\mathrm{s})}=\frac{\frac{\mathrm{V}_{\mathrm{o}}^{2}}{\mathrm{P}_{\mathrm{o}}} \cdot\left(\mathrm{s} \cdot \mathrm{R}_{\mathrm{SE}} \cdot \mathrm{C}_{\mathrm{o}}+1\right)}{\mathrm{s} \frac{\mathrm{C}_{\mathrm{o}} \cdot \mathrm{V}_{\mathrm{o}}^{2}}{\mathrm{P}_{\mathrm{o}}}\left(1+\frac{\mathrm{R}_{\mathrm{SE}} \cdot \mathrm{P}_{\mathrm{o}}}{\mathrm{V}_{\mathrm{o}}^{2}}\right)+1} \cdot \frac{3 \mathrm{~V}_{\mathrm{P}}}{2 \mathrm{~V}_{\mathrm{o}}}
$$

Logo:

$$
\frac{\mathrm{V}_{\mathrm{o}}(\mathrm{s})}{\mathrm{I}_{\mathrm{P}}(\mathrm{s})}=\frac{3 \cdot \mathrm{V}_{\mathrm{P}} \cdot \mathrm{V}_{\mathrm{o}}}{2 \cdot \mathrm{P}_{\mathrm{o}}} \cdot \frac{\mathrm{s} \cdot\left[\mathrm{R}_{\mathrm{SE}} \cdot \mathrm{C}_{\mathrm{o}}\right]+1}{\mathrm{~s} \cdot\left[\frac{\mathrm{C}_{\mathrm{o}} \cdot \mathrm{V}_{\mathrm{o}}^{2}}{\mathrm{P}_{\mathrm{o}}} \cdot\left(1+\frac{\mathrm{R}_{\mathrm{SE}} \cdot \mathrm{P}_{\mathrm{o}}}{\mathrm{V}_{\mathrm{o}}^{2}}\right)\right]+1}
$$

Desta forma, pode-se implementar três malhas de corrente independentes e uma única malha externa de tensão, onde a 
variável de controle da malha de tensão - $\mathrm{I}_{\mathrm{P}}(\mathrm{t})$ - é multiplicada pelas tensões de entrada, definindo então as referências para as malhas de corrente.

\section{IV - SNUBBER PASSIVO NÃO-DISSIPATIVO}

Em sua forma original, a topologia do retificador trifásico apresenta suas comutações dissipativas. Para elevar o rendimento da estrutura, um circuito de ajuda à comutação será introduzido nesta seção, representado para uma das fases do conversor. $\mathrm{O}$ circuito adotado possui características muito interessantes, já que é composto apenas por componentes passivos e permite o natural reaproveitamento da energia armazenada nos capacitores e indutores de comutação.

Observa-se na Fig. 13 que a fonte de entrada e o indutor foram representados por uma fonte de corrente, simplificação factível, pois a freqüência de comutação é muito maior do que a freqüência da rede de alimentação. A tensão de saída é considerada como uma fonte de tensão constante, todos os componentes são ideais e a representação feita é válida para todos os braços do retificador.

$\mathrm{O}$ capacitor $\mathrm{C}_{\mathrm{s} 11}$ auxilia durante o bloqueio do interruptor, controlando a derivada de crescimento da tensão. $\mathrm{O}$ indutor $\mathrm{L}_{\mathrm{s} 1}$ auxilia durante a entrada em condução do interruptor, permitindo que a tensão decresça até zero instantaneamente. $\mathrm{O}$ capacitor $\mathrm{C}_{\mathrm{s} 12}$ possui valor maior do que o capacitor $\mathrm{C}_{\mathrm{s} 11}$, permitindo, com o auxílio do indutor $\mathrm{L}_{\mathrm{s} 1}$, que a energia envolvida nas comutações seja entregue para a saída do retificador. Nota-se também, que as perdas por comutação do diodo $\mathrm{D}_{1}$ também são reduzidas com a utilização deste Snubber, pois a derivada de crescimento da tensão durante o bloqueio do mesmo pode ser controlada através dos capacitores auxiliares.

\section{$1^{\text {a }}$ etapa $\left(\mathbf{t}_{0}, \mathbf{t}_{1}\right)$}

Durante a primeira etapa o interruptor $\mathrm{S}_{1}$ está conduzindo, a tensão sobre o capacitor $\mathrm{C}_{\mathrm{s} 11}$ é nula e a tensão sobre o capacitor $\mathrm{C}_{\mathrm{s} 12}$ é $\mathrm{V}_{\mathrm{x}}$ (remanescente da última etapa). A Fig 13 apresenta esta etapa de funcionamento.

\section{$2^{\mathrm{a}}$ etapa $\left(\mathrm{t}_{1}, \mathrm{t}_{2}\right)$}

No instante $t_{1}$, o interruptor $S_{1}$ é comandado ao bloqueio, colocando em condução o diodo $\mathrm{D}_{\mathrm{s} 13}$. Assim, inicia-se a carga de forma linear do capacitor $\mathrm{C}_{\mathrm{s} 11}$. A Fig. 13 apresenta esta etapa de funcionamento.

\section{$3^{\text {a }}$ etapa $\left(t_{2}, t_{3}\right)$}

No instante $t_{2}$, o diodo $D_{\text {s11 }}$ entra em condução, iniciando a descarga de forma ressonante do capacitor $\mathrm{C}_{\mathrm{s} 12}$. O capacitor $\mathrm{C}_{\mathrm{s} 11}$ continua carregando-se, até atingir a tensão de saída $\mathrm{V}_{\mathrm{o}}$, dando início a próxima etapa de funcionamento. Esta etapa de funcionamento está representada na Fig. 13.

\section{4- etapa $\left(t_{3}, t_{4}\right)$}

Esta etapa de funcionamento é apresentada na Fig. 14. No instante $t_{3}, o$ diodo $D_{s 12}$ entra em condução. A tensão no capacitor $\mathrm{C}_{\mathrm{s} 12}$ e a corrente no indutor $\mathrm{L}_{\mathrm{s} 1}$ variam de forma ressonante. Esta etapa finda quando a tensão sobre o capacitor $\mathrm{C}_{\mathrm{s} 12}$ anula-se.

\section{5- etapa $\left(t_{4}, t_{5}\right)$}

Durante esta etapa de operação, a corrente que circula através do indutor $\mathrm{L}_{\mathrm{s} 1}$ cresce até atingir o valor da corrente $\mathrm{I}_{\mathrm{a}}$, dando início a próxima etapa. A Fig. 14 apresenta este estado topológico.

\section{$6^{\mathbf{a}}$ etapa $\left(t_{5}, t_{6}\right)$}

Durante esta etapa de operação não ocorre mudança em nenhuma variável de estado. Sendo que a duração desta etapa é dependente do comando dos interruptores, ou seja da razão cíclica. A representação desta etapa de funcionamento está apresentada na Fig. 14.

\section{$7^{\text {a }}$ etapa $\left(\mathbf{t}_{6}, \mathbf{t}_{7}\right)$}

Esta etapa de funcionamento tem início quando o interruptor $\mathrm{S}_{1}$ é comandado a conduzir. A tensão sobre o interruptor $S_{1}$ cai instantaneamente a zero, devido a presença do indutor $\mathrm{L}_{\mathrm{s} 1}$, propiciando sua comutação suave, uma vez que a corrente que circula através do mesmo cresce com derivada limitada por este indutor, transferindo-lhe a corrente que circula através do diodo $D_{1}$. A Fig. 15 mostra esta etapa de funcionamento, que finda quando o diodo $\mathrm{D}_{1}$ bloqueia-se.

\section{$8^{\text {a }}$ etapa $\left(t_{7}, t_{8}\right)$}

No instante $t_{7}$ a corrente no diodo $D_{1}$ é zero. $O$ capacitor $\mathrm{C}_{\mathrm{s} 11}$ descarrega-se enviando energia para o capacitor $\mathrm{C}_{\mathrm{s} 12} \mathrm{e}$ para o indutor $\mathrm{L}_{\mathrm{s} 1}$. A corrente que circula através dos elementos passivos varia de forma ressonante. Tem-se o término desta etapa quando a tensão sobre o capacitor $\mathrm{C}_{\mathrm{s} 11}$ torna-se nula. A Fig. 15 mostra esta etapa de funcionamento.

\section{9-a etapa $\left(t_{8}, t_{9}\right)$}

Durante a nona etapa de funcionamento a energia armazenada no indutor $\mathrm{L}_{\mathrm{s} 1}$ é transferida para o capacitor $\mathrm{C}_{\mathrm{s} 12}$. $\mathrm{O}$ término desta etapa ocorre quando a corrente no indutor $\mathrm{L}_{\mathrm{s} 1}$ anula-se. A Fig. 15 mostra esta etapa de funcionamento.

\section{Cálculo dos Elementos Passivos do Snubber}

A metodologia apresentada para o dimensionamento dos elementos passivos deste snubber é simplificada, entretanto um dimensionamento mais rigoroso pode ser encontrado em [8].

Com os valores de pico da corrente de entrada, a tensão média de saída e as derivadas de tensão e corrente calcula-se a indutância $\mathrm{L}_{\mathrm{s} 1}$ e as capacitâncias $\mathrm{C}_{\mathrm{s} 11}$ e $\mathrm{C}_{\mathrm{s} 12}$.

$$
\begin{aligned}
& \mathrm{L}_{\mathrm{s} 1}=\mathrm{V}_{\mathrm{o}} \cdot \frac{\mathrm{dt}}{\mathrm{di}} \\
& \mathrm{C}_{\mathrm{s} 11}=\mathrm{Ip} \cdot \frac{\mathrm{dt}}{\mathrm{dv}} \\
& \mathrm{C}_{\mathrm{s} 12}=4 \cdot \mathrm{C}_{\mathrm{s} 11}
\end{aligned}
$$



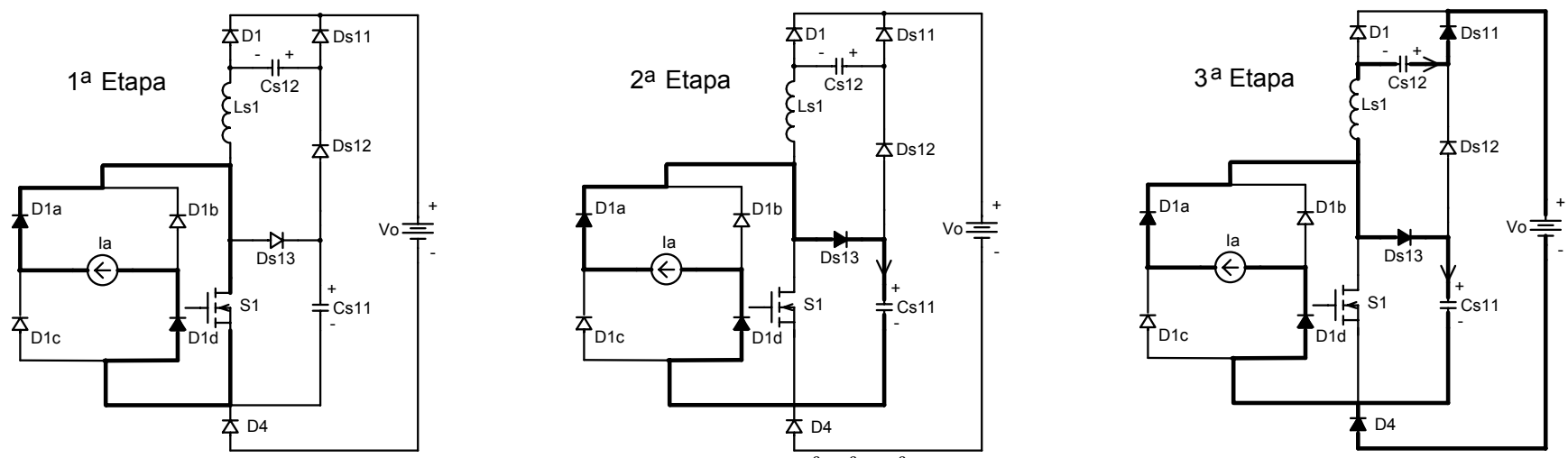

Fig. 13 - Circuitos equivalentes da $1^{\mathrm{a}}, 2^{\mathrm{a}}$ e $3^{\mathrm{a}}$ etapas de funcionamento.
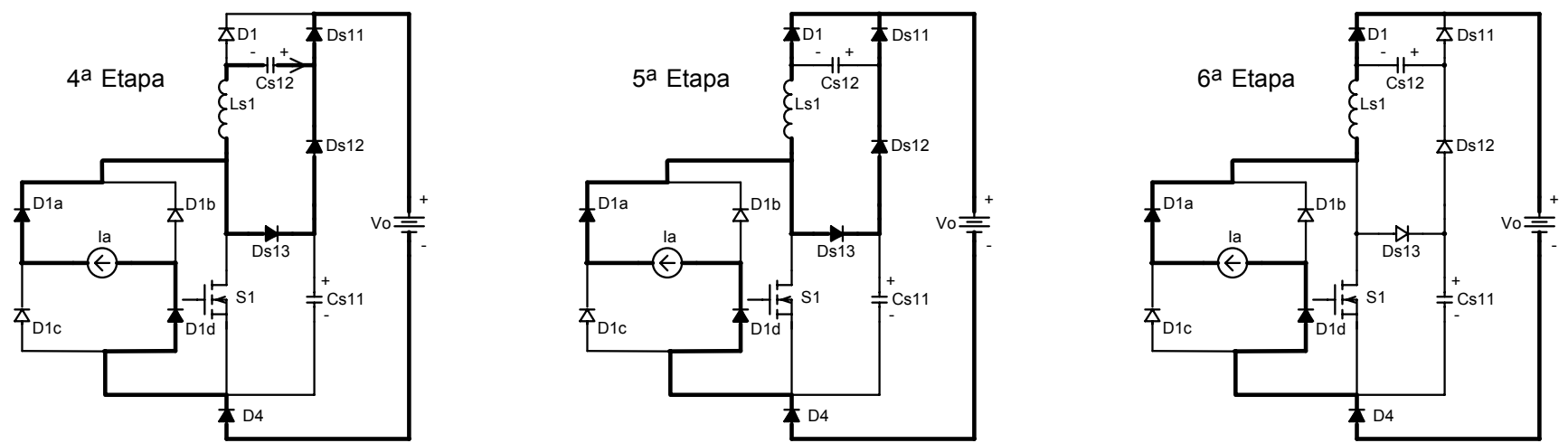

Fig. 14 - Circuitos equivalentes da $4^{\mathrm{a}}, 5^{\mathrm{a}}$ e $6^{\mathrm{a}}$ etapas de funcionamento.
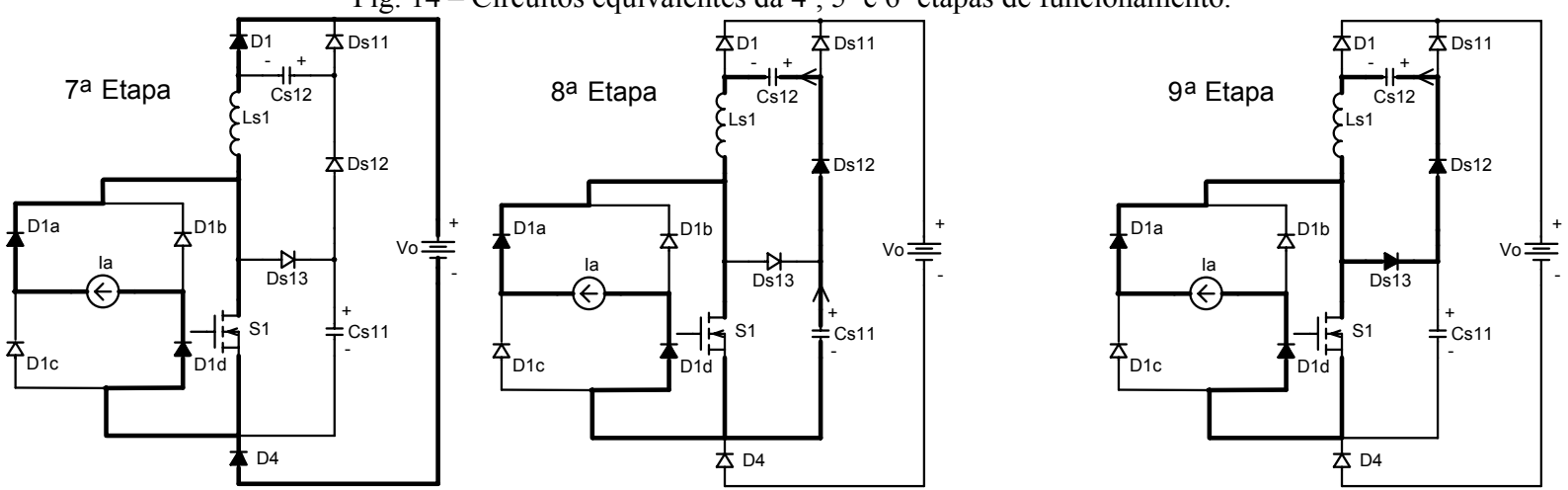

Fig. 15 - Circuitos equivalentes da $7^{\mathrm{a}}, 8^{\mathrm{a}}$ e $9^{\mathrm{a}}$ etapas de funcionamento.

\section{$\mathrm{V}$ - PROCEDIMENTO DE PROJETO E RESULTADOS EXPERIMENTAIS DA TOPOLOGIA 2 NÍVEIS}

\section{ESCOLHIDA}

Um protótipo da topologia dois níveis escolhida (Fig. 4) foi projetado, implementado e testado. Sejam as seguintes especificações do conversor:

$\mathrm{P}_{\mathrm{o}}=6 \mathrm{~kW} ; \mathrm{f}_{\text {rede }}=60 \mathrm{~Hz} ; \eta=\eta_{\text {ret }} \cdot \eta_{\mathrm{cc}-\mathrm{cc}}=0,88 ; \mathrm{f}_{\mathrm{s}}=50 \mathrm{kHz}$;

$\Delta \mathrm{i}_{\mathrm{a}, \mathrm{b}, \mathrm{c}_{\max }}=10 \% \mathrm{i}_{\mathrm{a}, \mathrm{b}, \mathrm{c}} ; \mathrm{V}_{\mathrm{L}}=220 \mathrm{~V}_{\mathrm{ef}}(+20 \%-30 \%)$

Onde $\mathrm{V}_{\mathrm{L}}$ é a tensão de linha eficaz nominal; $\Delta \mathrm{i}$, é o ripple relativo a corrente de pico máxima de entrada.

Para estipular o rendimento, considerou-se que um conversor CC-CC esteja conectado ao retificador e que o rendimento global seja de no mínimo $88 \%$, conforme os padrões Telebrás.

O valor de pico máximo e mínimo da tensão de linha e de fase é calculado como segue:

$$
\mathrm{V}_{\mathrm{L}_{\text {picomax }}}=\sqrt{2} \cdot \mathrm{V}_{\mathrm{L}} \cdot 1,2=\sqrt{2} \cdot 220 \cdot 1,20=373,4 \mathrm{~V}
$$

$$
\begin{gathered}
\mathrm{V}_{\mathrm{L}_{\text {picomin }_{\text {min }}}}=\sqrt{2} \cdot \mathrm{V}_{\mathrm{L}} 0,7=\sqrt{2} \cdot 220 \cdot 0,7=217,8 \mathrm{~V} \\
\mathrm{~V}_{\mathrm{f}_{\text {pico }_{\text {max }}}}=\frac{\mathrm{V}_{\mathrm{L}_{\text {picomax }_{\text {max }}}}}{\sqrt{3}}=\frac{373}{\sqrt{3}}=215 \mathrm{~V} \\
\mathrm{~V}_{\mathrm{f}_{\text {picom }}}=\frac{\mathrm{V}_{\mathrm{L}_{\text {pico }}}}{\sqrt{3}}=\frac{217}{\sqrt{3}}=125,7 \mathrm{~V}
\end{gathered}
$$

A tensão no barramento $\mathrm{CC}$ do retificador deve ser maior que o valor de pico máximo da tensão de linha. Então definise este valor $20 \%$ acima da tensão de linha máxima.

$$
\mathrm{V}_{\mathrm{o}}=\mathrm{V}_{\mathrm{L}_{\text {picomax }}} \cdot 1,2=373 \cdot 1,2 \cong 450 \mathrm{~V}
$$

A corrente de pico máxima é calculada a seguir, considerando que Po seja a potência na saída do suposto conversor CC-CC.

$$
\mathrm{I}_{\mathrm{a}, \mathrm{b}, \mathrm{c}_{\text {pico }} \text { max }}=\frac{2}{3} \cdot \frac{\mathrm{P}_{\mathrm{o}}}{\mathrm{V}_{\mathrm{f}_{\text {picom }}} \cdot \eta}=\frac{2}{3} \cdot \frac{6 \cdot 10^{3}}{125,7 \cdot 0,88}=36,16 \mathrm{~A}
$$


O valor mínimo da indutância de entrada do retificador, determinado para o setor de operação em que ocorre a maior ondulação pode ser calculado por (51).

$$
\mathrm{L}_{\mathrm{a}, \mathrm{b}, \mathrm{c}}=\frac{\mathrm{Vf}_{\text {pico }_{\min }}}{\mathrm{f}_{\mathrm{s}} \cdot \Delta \mathrm{i}_{\mathrm{a}, \mathrm{b}, \mathrm{c}}} \cdot\left(1-\frac{3}{2} \cdot \frac{\mathrm{Vf}_{\text {pico }_{\text {min }}}}{\mathrm{V}_{\mathrm{o}}}\right) \cong 400 \mu \mathrm{H}
$$

\section{A. Controle do Retificador}

A estratégia utilizada para controlar o retificador a dois níveis, aproxima-se muito do que é convencionalmente utilizado nos retificadores monofásicos. Isto é, foram utilizados três circuitos integrados UC3854, onde cada qual controla uma corrente de entrada. Apenas um dos circuitos integrados controla a tensão de saída do conversor, gerando a referência de corrente para os outros dois. Uma malha de feed-forward comum a todos também foi utilizada. As tensões de referência senoidais são obtidas da própria rede através de uma conexão $\Delta / \Delta$ de três transformadores, já que o neutro não se faz presente neste sistema.

\section{B. Projeto das Malhas de Corrente}

A fim de garantir a fidelidade do sinal e o isolamento do estágio de controle, sensores de efeito Hall foram utilizados para realizar a amostragem das correntes de entrada e da tensão de barramento. A Fig. 16 representa a estratégia de controle para uma das fases.

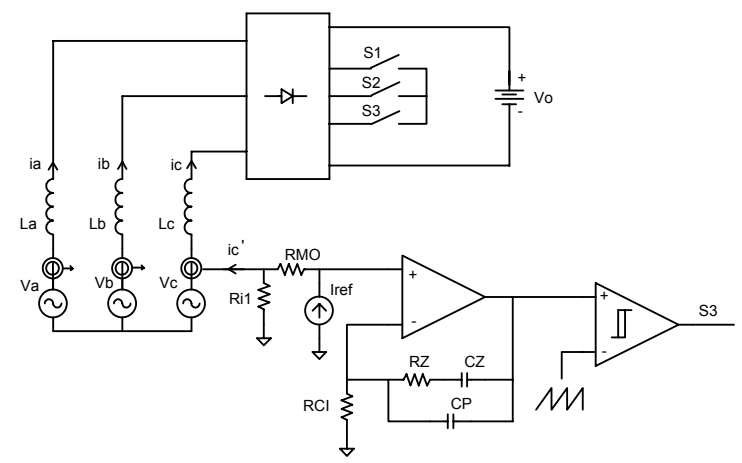

Fig. 16 - Estratégia de controle das correntes.

A função de transferência para o controle das correntes do retificador está apresentada em (52), onde $k_{i s}$ e $V_{T}$ são o ganho do sensor de corrente e o valor de pico da dente de serra do modulador, respectivamente.

$$
\begin{aligned}
\mathrm{G}_{\mathrm{i}}(\mathrm{s}) & =\frac{\Delta \mathrm{i}_{\mathrm{f}}(\mathrm{s})}{\Delta \mathrm{D}(\mathrm{s})}=\frac{2 \mathrm{~V}_{\mathrm{o}}}{\mathrm{s} 3 \mathrm{~L}_{\mathrm{a}, \mathrm{b}, \mathrm{c}}} \cdot \frac{\mathrm{k}_{\mathrm{is}}}{\mathrm{V}_{\mathrm{T}}} \\
\mathrm{G}_{\mathrm{i}}(\mathrm{s}) & =\frac{450}{\mathrm{~s} \cdot 3 \cdot 400 \cdot 10^{-6}} \cdot \frac{0,05}{5,2}
\end{aligned}
$$

Adotando-se uma valor comercial para $\mathrm{R}_{\mathrm{CI}}$, define-se $\mathrm{R}_{\mathrm{MO}}$, como segue:

$$
\mathrm{R}_{\mathrm{MO}}=\mathrm{R}_{\mathrm{CI}}=5,6 \mathrm{k} \Omega
$$

A fim de assegurar que o ganho na faixa plana do compensador seja de $20 \mathrm{~dB}$, determina-se $\mathrm{R}_{\mathrm{Z}}$.

$$
\mathrm{R}_{\mathrm{Z}}=10^{\frac{20}{20}} \cdot \mathrm{R}_{\mathrm{CI}}=56 \mathrm{k} \Omega
$$

$\mathrm{O}$ zero do controlador de corrente é posicionado em 2,8 $\mathrm{kHz}$. Assim, a capacitância $\mathrm{C}_{\mathrm{Z}}$ pode ser calculada conforme (56) e (57).

$$
\begin{gathered}
\mathrm{f}_{\mathrm{zi}}=2800 \mathrm{~Hz}=\frac{1}{2 \pi \mathrm{R}_{\mathrm{Z}} \mathrm{C}_{\mathrm{Z}}} \\
\mathrm{C}_{\mathrm{Z}}=\frac{1}{2800 \cdot 2 \cdot \pi \cdot 56 \cdot 10^{3}} \cong 1 \cdot 10^{-9} \mathrm{~F}
\end{gathered}
$$

O pólo do controlador de corrente é posicionado em 20 vezes a freqüência do zero, isto é $56 \mathrm{kHz}$. Assim, a capacitância $\mathrm{C}_{\mathrm{P}}$ é calculada em (58) e (59):

$$
\begin{gathered}
\mathrm{C}_{\mathrm{P}}=\frac{\mathrm{C}_{\mathrm{Z}}}{2 \cdot \pi \cdot \mathrm{fp}_{\mathrm{I}} \cdot \mathrm{R}_{\mathrm{Z}} \cdot \mathrm{C}_{\mathrm{Z}}-1} \\
\mathrm{C}_{\mathrm{P}}=\frac{1 \cdot 10^{-9}}{2 \cdot \pi \cdot 56000 \cdot 56000 \cdot 1 \cdot 10^{-9}-1} \cong 56 \cdot 10^{-12} \mathrm{~F}
\end{gathered}
$$

A função de transferência do controlador está representada por (60). A Fig. 17 mostra o diagrama de Bode de ganho das funções de transferência do conversor, controlador e FTLA . $_{i}$. O diagrama de Bode de fase está representado pela Fig. 18. Nota-se que a freqüência de cruzamento da FTLA $A_{i}$ ocorre em aproximadamente $1 / 5$ da freqüência de comutação, porém pode tornar-se necessário ajustá-la durante a experimentação.

$$
\mathrm{H}_{\mathrm{i}}(\mathrm{s})=\frac{\left(1+\mathrm{s} \cdot 56 \cdot 10^{-6}\right)}{\mathrm{s} \cdot 5,9 \cdot 10^{-6} \cdot\left(1+\mathrm{s} \cdot 2,97 \cdot 10^{-6}\right)}
$$

\section{Projeto da Malha de Tensão do Barramento CC}

Uma das principais vantagens da topologia utilizada como retificador dois níveis, foi a reduzida ondulação de baixa freqüência presente no barramento de saída do conversor, a qual idealmente é nula. Assim, não fosse a limitação tecnológica que restringe a corrente eficaz circulante nos capacitores de saída, poderiam ser utilizados valores reduzidos desta capacitância, o que propiciaria um controle relativamente mais rápido. Entretanto, foram necessários $3 \mathrm{mF}$ de capacitância, obtida através da associação (série/paralela) de capacitores de $1000 \mu \mathrm{F} / 250 \mathrm{~V}$. $\mathrm{O}$ controlador de tensão adotado foi o mesmo utilizado para o controle das correntes, ajustado de tal forma que a freqüência de cruzamento em laço aberto da planta ocorresse em $10 \mathrm{~Hz}$.

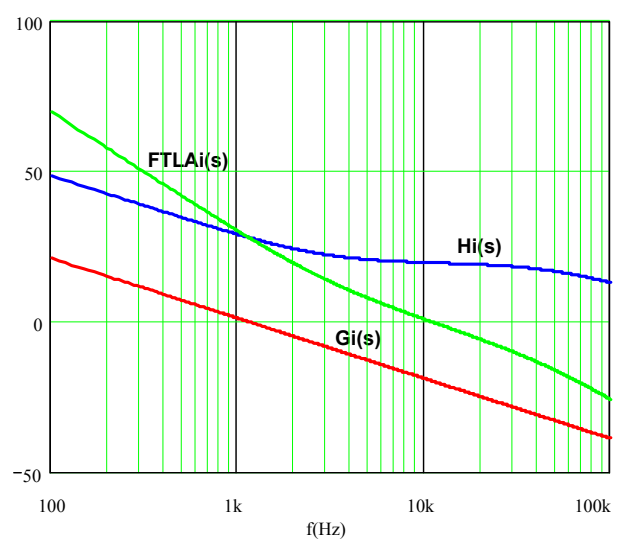

Fig. 17 - Diagrama de Bode de módulo de $\mathrm{G}_{\mathrm{i}}(\mathrm{s}), \mathrm{H}_{\mathrm{i}}(\mathrm{s})$ e FTLA $\mathrm{i}(\mathrm{s})$. 


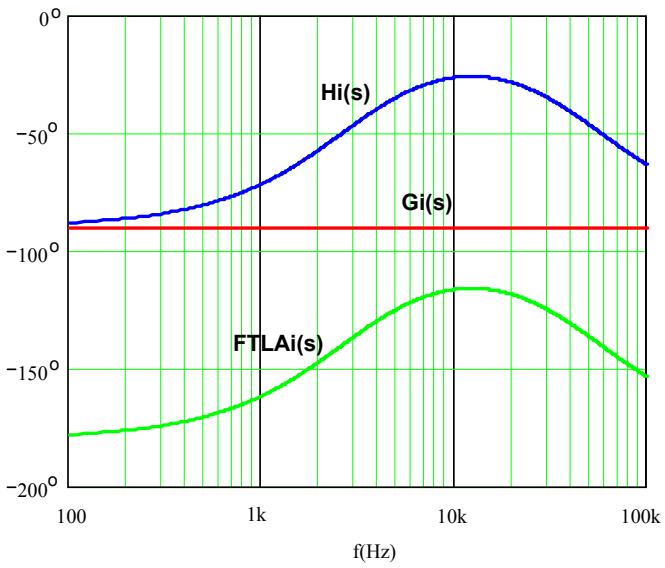

Fig. 18 - Diagrama de Bode de fase de $\mathrm{G}_{\mathrm{i}}(\mathrm{s}), \mathrm{H}_{\mathrm{i}}(\mathrm{s})$ e FTLA $_{\mathrm{i}}(\mathrm{s})$.

\section{Resultados Experimentais}

Os principais resultados experimentais obtidos para esta topologia são apresentados nesta seção. Os valores comerciais utilizados para implementação do Snubber foram os seguintes: $\mathrm{C}_{\mathrm{s} 11}=33 \mathrm{nF}, \mathrm{C}_{\mathrm{s} 12}=150 \mathrm{nF}, \mathrm{L}_{\mathrm{s} 1}=4,5 \mu \mathrm{H}$.

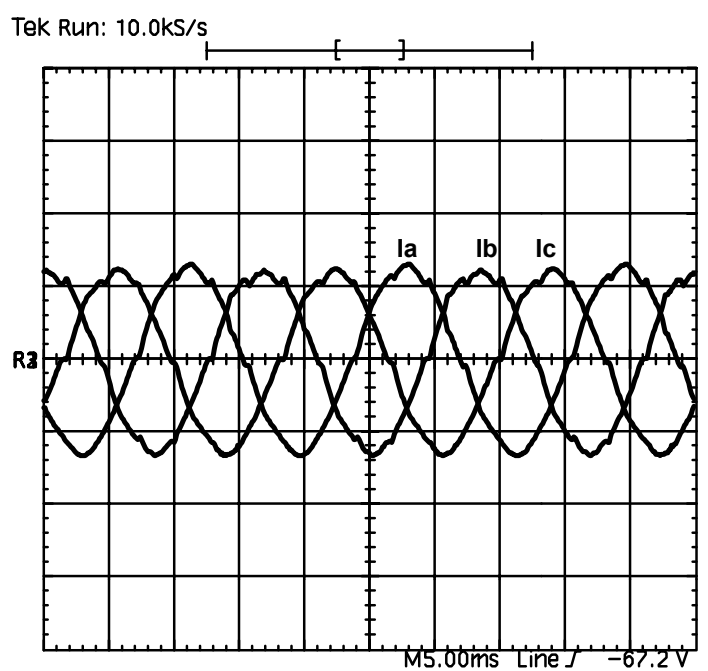

Fig. 19 - Corrente (20A/div.) das três fases do retificador.

Na Fig. 19 são apresentadas as correntes das três fases de entrada do retificador. Por inspeção visual, pode-se notar que os formatos são praticamente senoidais e defasados de $120^{\circ}$. Comprovando a característica de elevado fator de potência atribuída aos retificadores com controle ativo da corrente de entrada, mostra-se na Fig. 20 a tensão e a corrente em uma das fases. A análise harmônica destes resultados é apresentada na Tabela 1.

$\mathrm{Na}$ Fig. 21 tem-se um detalhe da tensão de saída e a corrente da fase "a" do retificador. Nesta, observa-se a reduzida ondulação de baixa freqüência na tensão de saída, o que reduz em muito os esforços de controle caso um segundo estágio seja conectado ao retificador, como em aplicações típicas de telecomunicações. $O$ transitório completo de partida do retificador trifásico é mostrado pela Fig. 22, em que são monitoradas a corrente de uma das fases e a tensão do barramento. Cabe observar que a pré-carga dos capacitores do barramento foi realizada pelo método convencional, utilizando-se resistores e contactores.

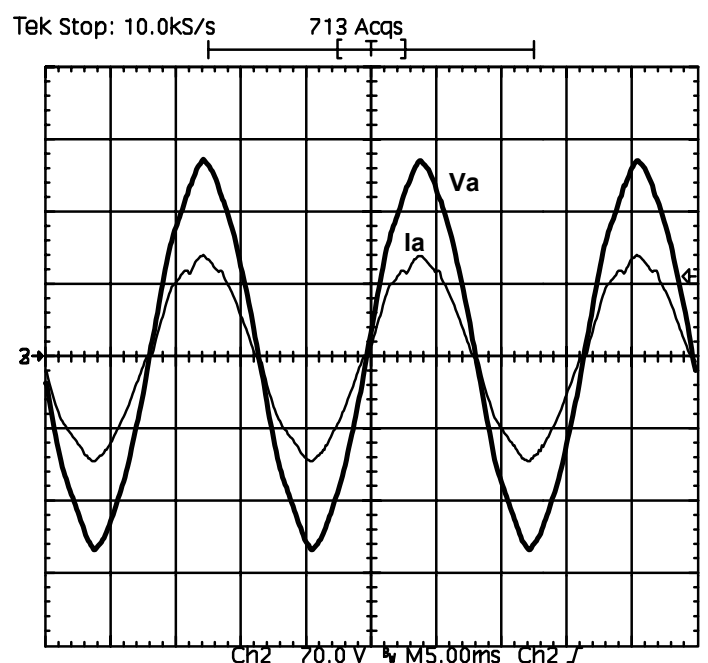

Fig. 20 - Tensão da fase 1 (70V/div) e corrente de entrada (20A/div).

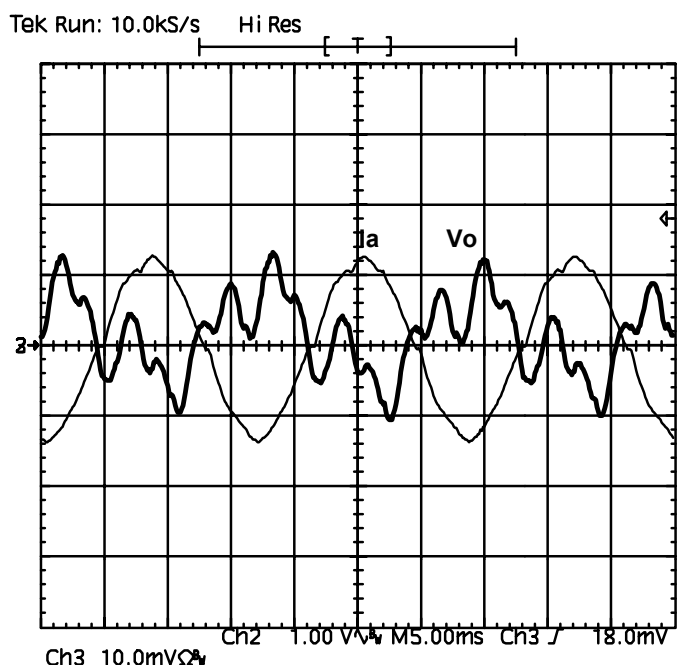

Fig. 21 - Detalhe da tensão de saída do retificador $1 \mathrm{~V} /$ div e corrente de uma das fases $20 \mathrm{~A} / \mathrm{div}$.

A tensão sobre um dos diodos do retificador não ultrapassa os $500 \mathrm{~V}$, garantindo uma boa margem de segurança para o equipamento projetado, conforme pode ser visto na Fig. 23. Na Fig. 24 apresenta-se a tensão sobre o interruptor MOSFET da fase "a" do retificador. Nas suas derivadas pode ser observada a influência do circuito de ajuda à comutação.

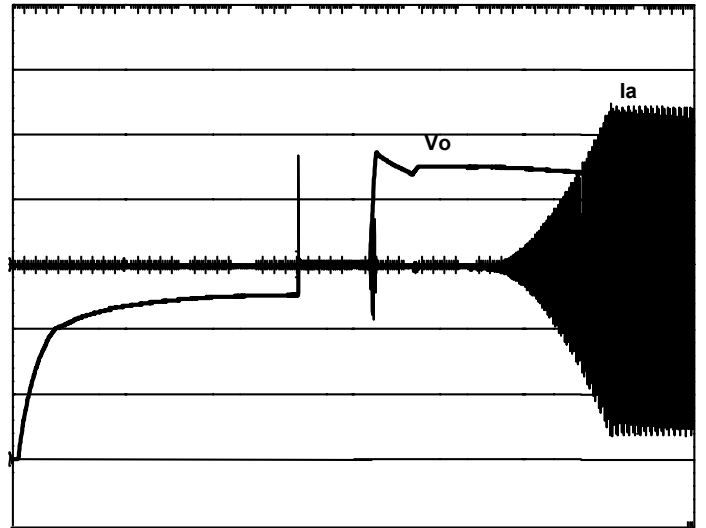

Fig. 22 - Transitório de partida do retificador. 


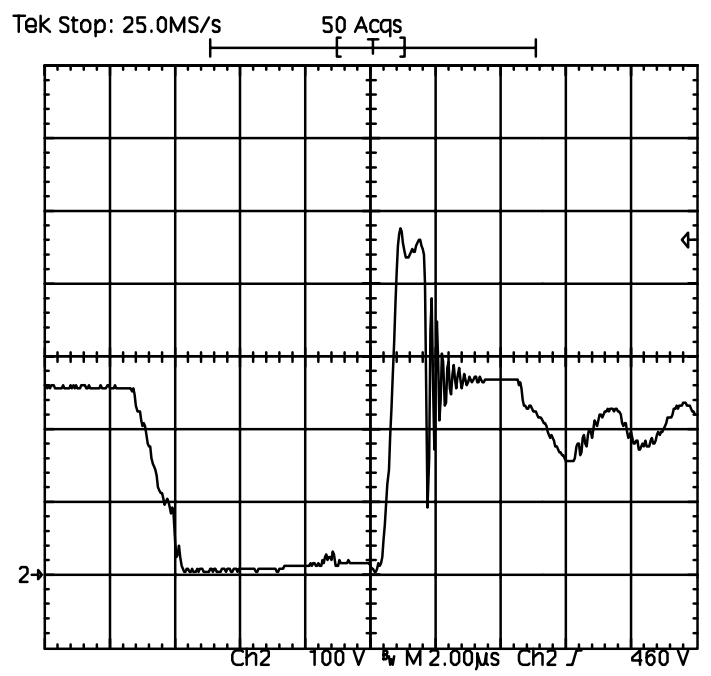

Fig. 23 - Tensão sobre o diodo $\mathrm{D}_{1}$ do retificador (com snubber).

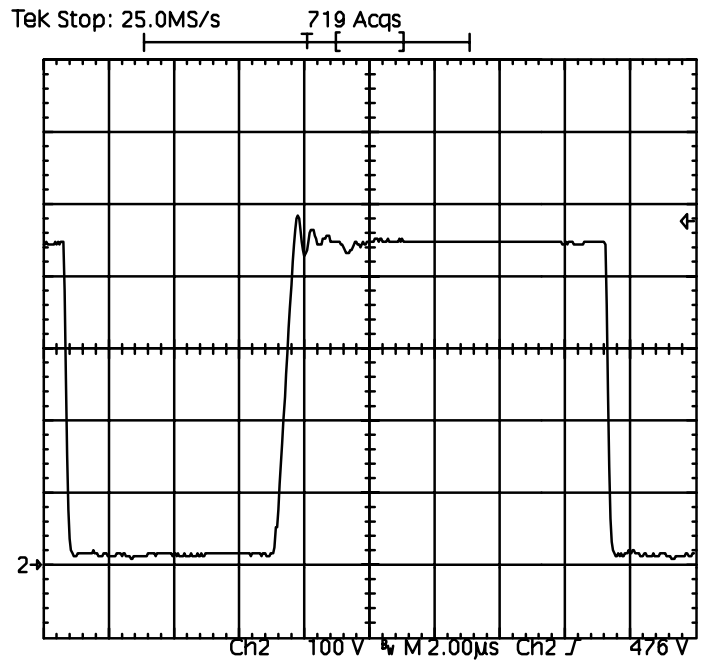

Fig. 24 - Tensão sobre um dos interruptores MOSFET do retificador.

Tabela 1: Taxa de Distorção Harmônica (THD) das tensões e correntes de entrada e fator de potência (FP).

\begin{tabular}{c|c|c|c}
\hline Fases & $\mathbf{T D H}_{\mathbf{V}}$ & $\mathbf{T D H}_{\mathbf{i}}$ & $\mathbf{F P}$ \\
\hline $\mathrm{a}$ & $2,57 \%$ & $5,04 \%$ & 0,995 \\
\hline $\mathrm{b}$ & $2,57 \%$ & $5,66 \%$ & 0,994 \\
\hline $\mathrm{c}$ & $4,70 \%$ & $5,68 \%$ & 0,992 \\
\hline
\end{tabular}

O comportamento do fator de potência do retificador frente a variações de carga é apresentado na Fig. 25, onde percebe-se os excelentes resultados obtidos.

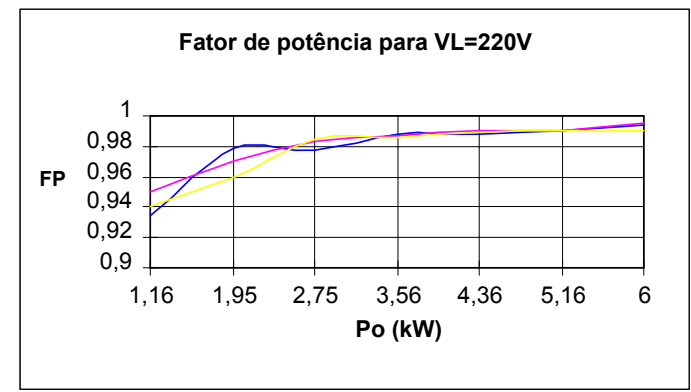

Fig. 25 -Curva de fator de potência das três fases do retificador.

As curvas de rendimento do conversor são mostradas na Fig. 27. Para as condições nominais de operação o rendimento do retificador situou-se em torno de 95,6\%, já para a menor tensão eficaz de entrada, o redimento teve uma redução de aproximadamente dois pontos percentuais.

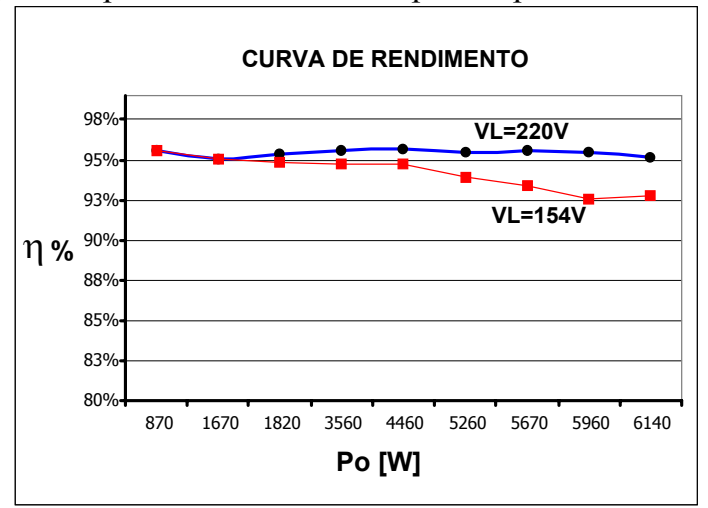

Fig. 26 - Curva de rendimento do retificador em função da potência de saída, tomando-se como parâmetro valores de tensão eficaz de entrada.

\section{VI - PROCEDIMENTO DE PROJETO E RESULTADOS EXPERIMENTAIS DA TOPOLOGIA 3 NÍVEIS ESCOLHIDA}

A topologia três níveis escolhida (Fig. 6) foi projetada e testada. As especificações são:

$\mathrm{P}_{\mathrm{o}}=26 \mathrm{~kW}, \mathrm{f}_{\text {rede }}=60 \mathrm{~Hz}, \eta_{\text {ret }}=0,96, \mathrm{f}_{\mathrm{s}}=70 \mathrm{kHz}$,

$\Delta \mathrm{i}_{\mathrm{a}, \mathrm{b}, \mathrm{c}_{\max }}=10 \% \mathrm{i}_{\mathrm{a}, \mathrm{b}, \mathrm{c}}, \mathrm{V}_{\mathrm{L}_{\max }}=530 \mathrm{~V}_{\mathrm{ef}}, \mathrm{V}_{\mathrm{L}_{\text {min }}}=320 \mathrm{~V}_{\mathrm{ef}}$

Onde $V_{L}$ é a tensão de linha eficaz e $\Delta \mathrm{i}$ é a ondulação relativa a corrente de pico máxima de entrada.

$\mathrm{O}$ valor de pico máximo e mínimo da tensão de linha e de fase é calculado como segue:

$$
\begin{aligned}
& \mathrm{V}_{\mathrm{L}_{\text {pico }_{\text {max }}}}=\sqrt{2} \cdot \mathrm{V}_{\mathrm{L}_{\text {max }}}=\sqrt{2} \cdot 530=750 \mathrm{~V} \\
& \mathrm{~V}_{\mathrm{L}_{\text {pico }_{\text {min }}}}=\sqrt{2} \cdot \mathrm{V}_{\mathrm{L}_{\text {min }}}=\sqrt{2} \cdot 320=453 \mathrm{~V} \\
& \mathrm{~V}_{\mathrm{f}_{\text {pico max }}}=\frac{\mathrm{V}_{\mathrm{L}_{\text {pico }} \text { max }}}{\sqrt{3}}=\frac{750}{\sqrt{3}}=433 \mathrm{~V} \\
& \mathrm{~V}_{f_{\text {pico }} \text { min }}=\frac{\mathrm{V}_{\mathrm{L}_{\text {pico }}}}{\sqrt{3}}=\frac{453}{\sqrt{3}}=262 \mathrm{~V}
\end{aligned}
$$

A tensão no barramento $\mathrm{CC}$ do retificador deve ser maior que o valor de pico máximo da tensão de linha. A tensão de barramento é então definida $20 \%$ acima da tensão de linha máxima.

$$
\mathrm{V}_{\mathrm{o}}=\mathrm{V}_{\mathrm{L}_{\text {pico }} \text { max }} \cdot 1,2=750 \cdot 1,2=900 \mathrm{~V}
$$

As correntes de pico máxima e minima são calculadas a seguir:

$$
\begin{aligned}
& \mathrm{I}_{\mathrm{a}, \mathrm{b}, \mathrm{c}_{\text {pico }} \text { max }}=\frac{2}{3} \cdot \frac{\mathrm{P}_{\mathrm{i}}}{\mathrm{V}_{\mathrm{f}_{\text {pico }}}}=\frac{2}{3} \cdot \frac{26 \times 10^{3} / 0,96}{262}=69 \mathrm{~A} \\
& \mathrm{I}_{\mathrm{a}, \mathrm{b}, \mathrm{c}_{\text {pico }}}=\frac{2}{3} \cdot \frac{\mathrm{P}_{\mathrm{i}}}{\mathrm{V}_{\mathrm{f}_{\text {pico }}}}=\frac{2}{3} \cdot \frac{26 \times 10^{3} / 0,96}{433}=42 \mathrm{~A}
\end{aligned}
$$

O ganho $\beta$ é calculado de acordo com a equação (67) e a indutância Boost de acordo com a equação (68).

$$
\beta=\frac{\mathrm{V}_{\mathrm{o}} / 2}{\mathrm{~V}_{\mathrm{f}_{\text {pico }} \text { min }}}=\frac{450}{262}=1,72
$$




$$
\mathrm{L}_{\mathrm{a}, \mathrm{b}, \mathrm{c}}=\frac{\mathrm{V}_{\mathrm{o}} / 2}{\Delta \mathrm{I}_{\mathrm{a}, \mathrm{b}, \mathrm{c}_{\max }} \mathrm{f}_{\mathrm{s}}} \cdot\left(\frac{1}{\beta}-\frac{3}{4 \cdot \beta^{2}}\right) \cong 400 \cdot 10^{-6} \mathrm{H}
$$

\section{A. Controle do Retificador}

$\mathrm{O}$ retificador a três níveis também é controlado utilizando-se três circuitos integrados do tipo UC3854B, sendo um para cada fase.

O sinal de sincronismo para a geração da corrente de referência é obtido através de três transformadores monofásicos ligados em $\Delta / \Delta$. A tensão do barramento CC é controlada pelo 3854 da fase "a", que também apresenta a malha de feed-forward. O sinal de saída do controlador de tensão e da malha de feed-forward é então entregue aos CIs da fase "b" e "c" para a geração das correntes de referência.

\section{B. Projeto das Malhas de Corrente}

Assim como no retificador a 2 níveis, para o retificador a 3 níveis também é necessário se utilizar sensores de efeito Hall para monitorar as correntes de entrada e as tensões no barramento $\mathrm{CC}$, garantindo o isolamento do estágio de controle.

A função de transferência $G_{i}(s)$ é calculada de acordo com a equação (69).

$$
\mathrm{G}_{\mathrm{i}}(\mathrm{s})=\frac{\Delta \mathrm{i}_{\mathrm{f}}(\mathrm{s})}{\Delta \mathrm{D}(\mathrm{s})}=\frac{\mathrm{V}_{\mathrm{o}}}{\mathrm{s} 3 \mathrm{~L}_{\mathrm{a}, \mathrm{b}, \mathrm{c}}}=\frac{900}{\mathrm{~s} \cdot 3 \cdot 400 \cdot 10^{-6}}=\frac{750 \cdot 10^{3}}{\mathrm{~s}}
$$

Os resistores $R_{M O}$, e $R_{C I}$ e $R_{z}$ são calculados como mostrado a seguir, sendo que $K_{i}$ é o ganho de amostra da corrente de entrada:

$$
\begin{aligned}
& \mathrm{R}_{\mathrm{MO}}=\mathrm{R}_{\mathrm{CI}}=\frac{\mathrm{i}_{\mathrm{a}, \mathrm{b}, \mathrm{c} \text { limite }} \mathrm{K}_{\mathrm{i}}}{\mathrm{i}_{\text {mult.max }}}=\frac{116,45 \cdot 60 \cdot 10^{-3}}{281,4 \cdot 10^{-6}} \cong 22 \mathrm{k} \Omega \\
& \mathrm{R}_{\mathrm{z}}=10^{\frac{18}{20}} \cdot \mathrm{R}_{\mathrm{CI}} \cong 180 \mathrm{k} \Omega
\end{aligned}
$$

$\mathrm{O}$ zero do controlador de corrente é posicionado em $4 \mathrm{kHz}$. Assim, a capacitância $\mathrm{C}_{\mathrm{z}}$ é calculada:

$$
\begin{aligned}
& \mathrm{f}_{\mathrm{zi}}=4000 \mathrm{~Hz}=\frac{1}{2 \pi \mathrm{R}_{\mathrm{z}} \mathrm{C}_{\mathrm{z}}} \\
& \mathrm{C}_{\mathrm{z}}=\frac{1}{4000 \cdot 2 \cdot \pi \cdot 180 \cdot 10^{3}} \cong 220 \cdot 10^{-12} \mathrm{~F}
\end{aligned}
$$

$\mathrm{O}$ pólo do controlador de corrente é posicionado em 32kHz. Assim, a capacitância $\mathrm{C}_{\mathrm{p}}$ é calculada:

$$
\begin{aligned}
& \mathrm{f}_{\mathrm{pi}}=32000 \mathrm{~Hz}=\frac{\mathrm{C}_{\mathrm{z}}+\mathrm{C}_{\mathrm{p}}}{2 \pi \mathrm{R}_{\mathrm{z}} \mathrm{C}_{\mathrm{z}} \mathrm{C}_{\mathrm{p}}} \\
& \mathrm{C}_{\mathrm{p}}=\frac{220 \cdot 10^{-12}}{32000 \cdot 2 \cdot \pi \cdot 180 \cdot 10^{3} \cdot 220 \cdot 10^{-12}-1} \cong 33 \cdot 10^{-12} \mathrm{~F}
\end{aligned}
$$

A função de transferência do controlador e a função de transferência de laço aberto são apresentadas em (76) e (77), sendo $\mathrm{V}_{\mathrm{T}}$ o valor de pico do sinal dente de serra do modulador.

$$
\begin{aligned}
& \mathrm{H}_{\mathrm{i}}(\mathrm{s})=\frac{\left(1+\mathrm{s} \cdot 39,6 \cdot 10^{-6}\right)}{\mathrm{s} \cdot 5,6 \cdot 10^{-6} \cdot\left(1+\mathrm{s} \cdot 5,1 \cdot 10^{-6}\right)} \\
& \text { FTLA }_{\mathrm{i}}(\mathrm{s})=\frac{\mathrm{K}_{\mathrm{i}}}{\mathrm{V}_{\mathrm{T}}} \cdot \mathrm{G}_{\mathrm{i}}(\mathrm{s}) \cdot \mathrm{H}_{\mathrm{i}}(\mathrm{s})
\end{aligned}
$$

A Fig. 27 mostra o diagrama de Bode de módulo e a Fig. 28 o diagrama de Bode de fase da função de transferência do conversor, do controlador e da função de transferência de laço aberto. A freqüência de cruzamento da FTLA $_{i}$ ficou em $10 \mathrm{kHz}$.

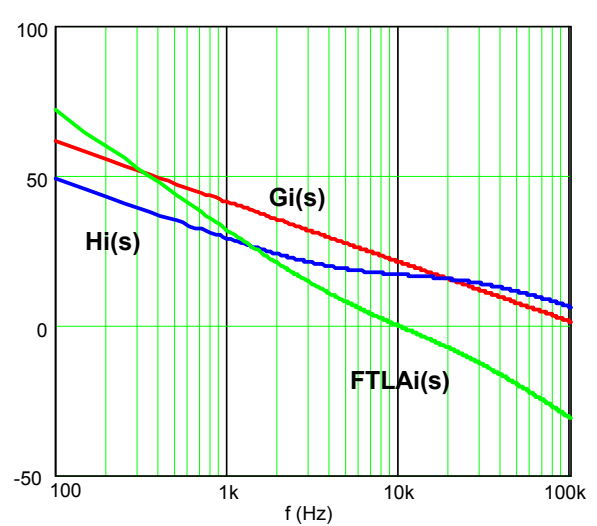

Fig. 27 - Diagrama de Bode de módulo de $\mathrm{G}_{\mathrm{i}}(\mathrm{s}), \mathrm{H}_{\mathrm{i}}(\mathrm{s})$ e FTLA $\mathrm{F}_{\mathrm{i}}(\mathrm{s})$.

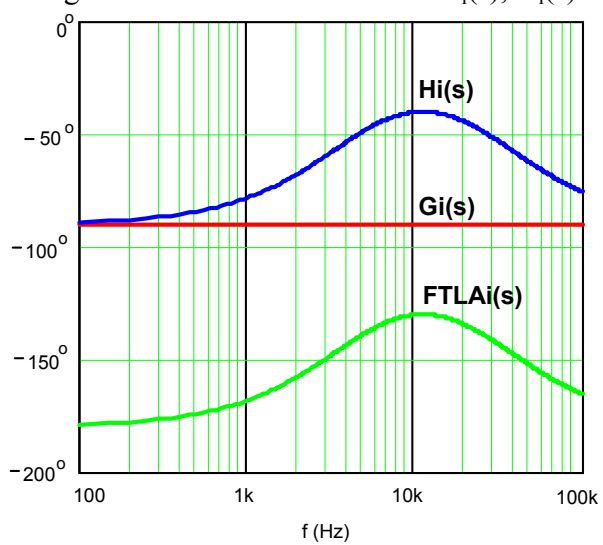

Fig. 28 - Diagrama de Bode de fase de $\mathrm{G}_{\mathrm{i}}(\mathrm{s}), \mathrm{H}_{\mathrm{i}}(\mathrm{s})$ e $\mathrm{FTLA}_{\mathrm{i}}(\mathrm{s})$.

\section{Projeto da Malha de Tensão do Barramento CC}

Para compor os capacitores de saída é utilizada uma associação série/paralelo de capacitores de $10 \mathrm{mF} / 250 \mathrm{~V}$ da Siemens (B43875), resultando em um capacitor total de $5 \mathrm{mF} / 1000 \mathrm{~V}$.

O controlador adotado foi o mesmo utilizado para as malhas de corrente e foi ajustado de tal forma que a freqüência de cruzamento de ganho da $\mathrm{FTLA}_{\mathrm{v}}$ ocorresse em aproximadamente $10 \mathrm{~Hz}$.

\section{Projeto da Malha de Balanceamento das Tensões no Barramento CC}

O balanceamento das tensões dos capacitores do barramento CC é fundamental para que se garanta que todos os semicondutores fiquem submetidos à metade da tensão de barramento. Esta malha já possui um integrador e portanto, um controlador do tipo proporcional é utilizado. O ganho do controlador foi ajustado por simulação e no laboratório.

\section{E. Resultados Experimentais}

Os resultados experimentais do retificador 3 níveis são apresentados nesta seção. O circuito Snubber foi projetado de acordo com [8], resultando em $\mathrm{C}_{\mathrm{s} 11}=68 \mathrm{nF}, \mathrm{C}_{\mathrm{s} 12}=330 \mathrm{nF}$ e $\mathrm{L}_{\mathrm{s} 1}=2 \mu \mathrm{F}$.

Os resultados apresentados nas Figs. 30 a 32 são para a tensão mínima de entrada $\left(\mathrm{V}_{\mathrm{f}_{\text {picomin }}}=262 \mathrm{~V}\right)$. Na Fig. 29 são apresentadas a tensão da rede na fase "a" e as correntes nas três fases. Pode-se obervar o elevado fator de potência e a defasagem de $120^{\circ}$ entre as correntes. A análise harmônica das tensões e correntes de entrada é apresentada na Tabela 2, estando em conformidade com a norma IEC-61000-3-4. 


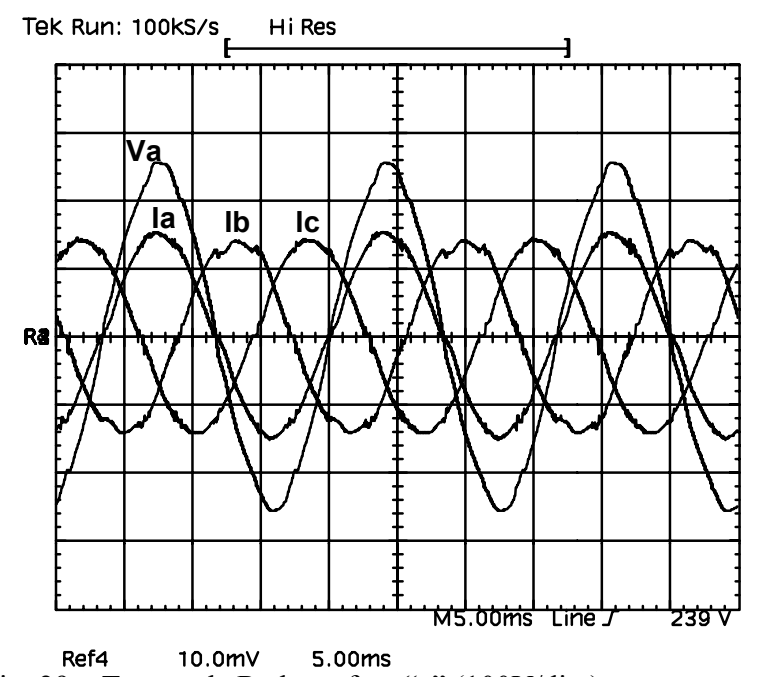

Fig. 29 - Tensão da Rede na fase "a" (100V/div.) e correntes de entrada nas três fases (50A/div.).

Na Fig. 30 pode-se observar a tensão sobre o MOSFET $\mathrm{S}_{1 \mathrm{a}}$ da fase "a", na qual verifica-se o controle das derivadas de tensão proporcionado pelo circuito Snubber.

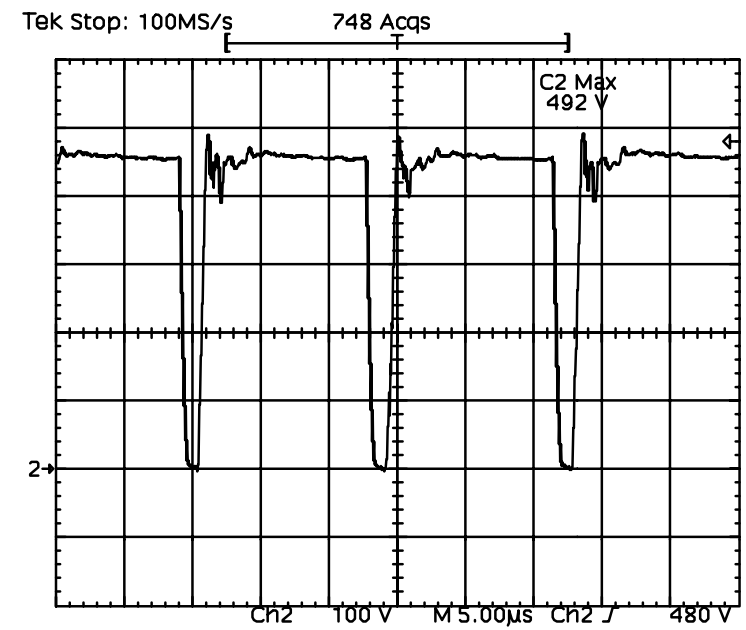

Fig. 30 - Tensão na chave $\mathrm{S}_{1 \mathrm{a}}(100 \mathrm{~V} / \mathrm{div}$.).

Na Fig. 31 verifica-se que a tensão sobre o diodo Boost $D_{1}$ é maior que a metade da tensão de barramento. Isto acontece apenas nos diodos Boost $\left(\mathrm{D}_{1}, \mathrm{D}_{2}, \mathrm{D}_{3}, \mathrm{D}_{4}, \mathrm{D}_{5}\right.$ e $\left.\mathrm{D}_{6}\right)$ pois a metade da tensão de barramento é somada a tensão no capacitor $\mathrm{C}_{\mathrm{s} 12}$, ao final da etapa 4 de operação do Snubber.

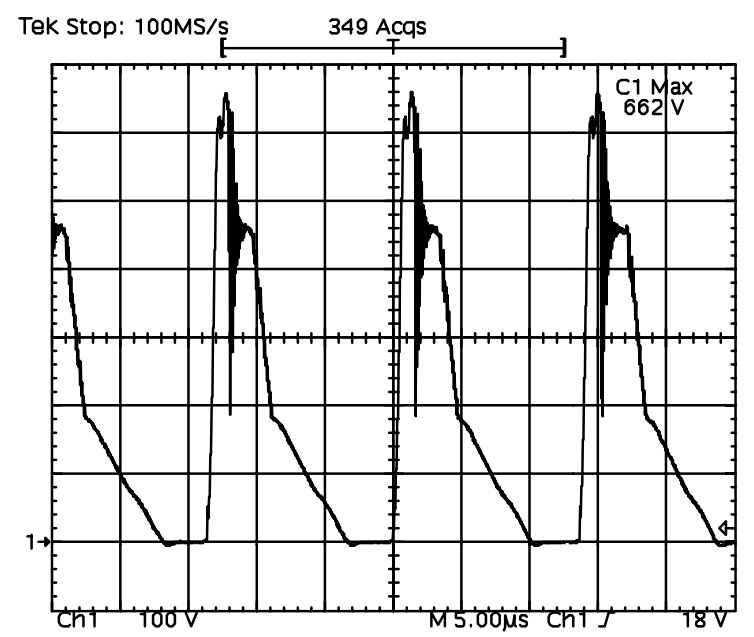

Fig. 31 - Tensão no Diodo Boost $\mathrm{D}_{1}(100 \mathrm{~V} /$ div. $)$.
Tabela 2: distorção harmônica das tensões e correntes de entrada e fator de potência.

\begin{tabular}{c|c|c|c}
\hline Fases & $\mathrm{TDH}_{\mathrm{V}}$ & $\mathrm{TDH}_{\mathrm{i}}$ & $\mathrm{FP}$ \\
\hline $\mathrm{a}$ & $2,45 \%$ & $5,33 \%$ & 0,992 \\
\hline $\mathrm{b}$ & $2,50 \%$ & $5,12 \%$ & 0,998 \\
\hline $\mathrm{c}$ & $2,85 \%$ & $8,36 \%$ & 0,995 \\
\hline
\end{tabular}

Na Fig. 32 mostra-se o transitório completo de partida do retificador, apresentando-se a tensão em cada um dos capacitores do barramento e a corrente em uma das fases. A pré-carga dos capacitores do barramento é realizada com resistores e contactores. O retificador parte a vazio e após a estabilização das tensão de barramento, pode-se colocar carga.

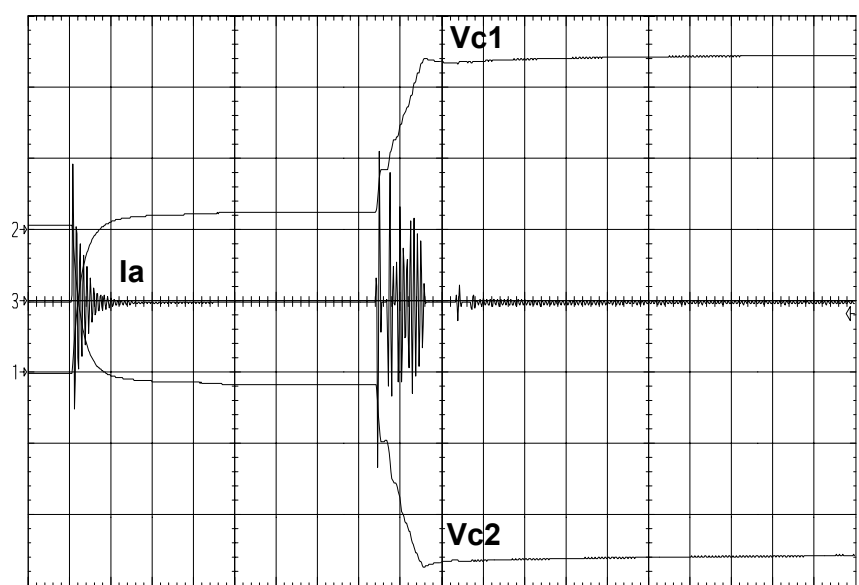

Fig. 32 - Transitório de partida do retificador (100V/div., 20A/div.).

A curva de rendimento do retificador para a tensão mínima de entrada é apresentada na Fig. 33. O rendimento para tensão máxima não foi medido, mas deve ser superior, pois as correntes são menores.

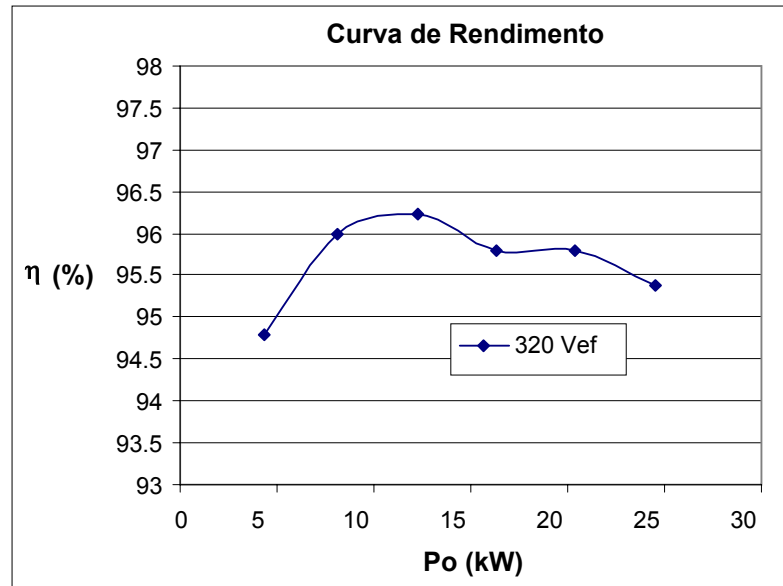

Fig. 33 - Curva de rendimento do retificador em função da potência, para a tensão mínima de entrada.

\section{CONCLUSÃO}

Foram apresentadas algumas das principais topologias encontradas na literatura, para conversores CA-CC PWM trifásicos, dois e três níveis, dentre as quais foram selecionadas duas topologias, uma em 2 e outra em 3 níveis. Os principais critérios utilizados na escolha destas topologias foram: simplicidade, menor quantidade de componentes e menores esforços. 
$\mathrm{Na}$ sequência foi apresentada uma modelagem genérica para estes conversores, apresentando-se um modelo completo e outro simplificado, sendo utilizado este último para projetar os controladores.

Foi apresentado ainda um Snubber passivo não dissipativo, para garantir a comutação suave dos interruptores, reduzindo as perdas por comutação.

Finalmente, apresenta-se o projeto e os resultados experimentais obtidos, a partir de dois protótipos implementados, um 2 níveis de $6 \mathrm{~kW}$ e outro 3 níveis de $26 \mathrm{~kW}$.

Observou-se uma pequena distorção nas correntes de entrada obtidas experimentalmente, em relação às observadas em simulação. Isto se deve principalmente às distorções das tensões da rede de alimentação, combinada ao fato de se ter utilizado o modelo simplificado do conversor, de forma que os controladores não agem de forma independente, mas interagem entre si. Mesmo assim, ambos os resultados atendem a norma IEC -61000-3-4.

\section{REFERÊNCIAS BIBLIOGRÁFICAS}

[1] N. Mohan, T. M. Undeland, W. P. Robbins. "Power Electronics: Converters, Applications, and Design", John Wiley \& Sons, 1989.

[2] T. Salzmann and A. Weschta, "Progress in voltage source inverters and current source inverters", Conf. Rec. of IEEE-IAS Annual Meeting, pp. 577-583, 1987.

[3] A. Nabae, I. Takahashi, and H. Akagi, "A new neutral-point-clamped PWM inverter”, IEEE Trans. Ind. Appl., vol 17, no. 5, pp.518-523, Sept/Octo, 1981.

[4] G. Spiazzi, and F. C. Lee, "Implementation of single-phase boost power factor correction circuits in three-phase applications", Switching Rectifiers for Power Factor Correction, Volume V, VPEC Publication Series.

[5] Y. Zhao, Y. Li , and T. A. Lipo, "Force Commutated Three Level Boost Type Rectifier", IEEE Trans. Ind. Appl., vol. 31, no. 1, January/February 1995

[6] J. W. Kolar, and F. C. Zach, "A novel three-phase utility interface minimizing line current harmonics of high-power telecommunications", Proc. of IEEE Int. Telecommunications Energy Conf., pp. 367-374, 1994.

[7] J. W. Kolar, F. C. Zach, “A Novel Three-Phase Three Level Unity Power Factor PWM Rectifier", 28 ${ }^{\text {th }}$ Power Conversion Conference, Nüremberg, Germany, June 28-30, 1994, pp. 125-138.

[8] A. C. C. Neto, "Retificador PWM Trifásico de 26 kW, Três Níveis, Unidirecional, Fator de Potência Unitário e Alto Rendimento para Aplicação em Centrais de Telecomunicação", Dissertação de Mestrado, INEP/EEL/UFSC, Abril/2002.

[9] D. Borgonovo, "Modelagem e Controle de Retificadores PWM Trifásicos Empregando a Transformação de Park", Dissertação de Mestrado, INEP/EEL/UFSC, Novembro/2001.

[10] V. Vorpérian, "Simplified Analysis of PWM Converters Using the Model of the PWM Switch: Parts I and II", IEEE Trans. Aerospace and Electronic Systems 26, May 1990, pp. 490-505.

\section{DADOS BIOGRÁFICOS}

Ivo Barbi recebeu os graus de Engenheiro Eletricista e Mestre em Engenharia Elétrica em 1973 e 1976, respectivamente, pela Universidade Federal de Santa Catarina, e o grau de Doutor em Engenharia pelo Institut National Polytechnique de Toulose, França, em 1979. Ivo Barbi fundou a Sociedade Brasileira de Eletrônica de Potência (SOBRAEP) e o Instituto de Eletrônica de Potência da Universidade Federal de Santa Catarina. Atualmente ele é Professor Titular em Eletrônica de Potência. Desde 1992 ele é Editor Associado na área de Conversores de Potência da IEEE Transactions on Industrial Electronics. Seus campos de interesse são conversores estáticos para alta freqüência e alto rendimento e correção do fator de potência de fontes de alimentação.

Yales Rômulo Novaes, Nasceu em Indaial - SC, concluiu o curso em Engenharia Elétrica Industrial pela FURB Universidade Regional de Blumenau em 1998 e o mestrado no Instituto de Eletrônica de Potência - INEP na Universidade Federal de Santa Catarina em 2000. Entre 2000 e 2001 trabalhou como engenheiro de desenvolvimento no INEP onde atualmente está realizando seu doutoramento em eletrônica de potência, pesquisando na área de células à combustível. Suas áreas de interesse são: células a combustível, retificadores com alto fator de potência, inversores, comutação suave e conversores para telecomunicações.

Fabiana Pöttker de Souza recebeu os graus de Engenheira Eletricista, Mestre e Doutora em Engenharia Elétrica em 1995, 1997 e em 2000, respectivamente, pela Universidade Federal de Santa Catarina. Atualmente é Professora substituta na Universidade Federal de Santa Catarina (UFSC) e Engenheira de Desenvolvimento no INEP-UFSC. Suas áreas de interesse incluem correção do fator de potência de fontes de alimentação e filtros ativos.

Deivis Borgonovo Nasceu em Rio do Sul - SC em 1977, recebeu os graus de Engenheiro Eletricista e Mestre em Engenharia Elétrica em 1999 e 2001 respectivamente, pela Universidade Federal de Santa Catarina - UFSC -. Atualmente é doutorando no Instituto de Eletrônica de Potência - INEP - na UFSC. Suas áreas de interesse incluem correção de fator de potência, retificadores trifásicos com alto fator de potência e controle de conversores estáticos. 